\title{
Effect of Growing Media and Nutrient Solutions by Using Drip Hydroponic System on Growth, Flowering and Quality of Lilium bulbs
}

\author{
${ }^{1}$ Taghreed E. Eissa, ${ }^{2}$ Magdy A. Eskarous and ${ }^{2}$ Enga M. Niel
}

${ }^{I}$ Ornamental Plants and Landscape Gardening Res. Dept., Hort. Res. Inst., ARC, Giza, Egypt. ${ }^{2}$ Soil Fertility and Plant Nutrition Dept., Water and Environment Res. Inst., ARC, Giza, Egypt.

Received: 12 Sept. 2019 / Accepted 05 Nov. 2019 / Publication date: 20 Nov. 2019

\begin{abstract}
This study was administrated to analyze the impact of drip hydroponic system on flowers and daughter bulblets production of the Asiatic lily hybrid plant cv. "Honesty" using some nutrient solutions (Hoagland No. 2 basal salt mixture, Murashige and Skoog basal salt mixture, algae extract (balanced fertilizer), Hoagland No. 2 basal salt mixture and balanced fertilizer, Hoagland No. 2 basal salt mixture and silicon, in addition to tap-water as a control) with gravels, vermiculite and organic soil conditioner (Hundz-soil) as media. Results showed that the drip hydroponic system was a good methodology to induce flowers in fifty days. Hoagland solution with Hundz-soil gave the best vegetative growth, flowering and bulblets production.
\end{abstract}

Keywords: Asiatic lily plant, hydroponic, Hundz-soil, nutrient solutions, flowering, daughter bulblets.

Abbreviations: Hoagland No. 2 basal salt mixture solution (Hoag S.); Murashige and Skoog basal salt mixture solution (MS); B.F. (Balanced fertilizer); silicon (Si) and EC, electrical conductivity.

\section{Introduction}

Lilies are one of the most necessary bulbous plants for production as cut flowers, for pot plant purposes and for parks, gardens and landscape decoration, Asiatic hybrid lilies blooms are larger than traditional. Their vase life is the longest of any liliaceous plant, and they boost the widest range of colors. Soil-less cultures are with success.

Using of many decades with the aim to attenuate production and reduce cost, or defined as the science of growing plants using a solution of appropriate nutrients rather than soil. The growing medium, acts as a support for the plants and their root systems, whereas the solution passes freely. Hydroponics may be the foremost intensive methodology of crop production in today's agricultural industry (Maloupa et al., 1993).

Drip systems are one of the most widely used types of hydroponic systems around the world, both for home growers as well as commercial growers. The way a drip system works is simply drip nutrient solution on the plant roots to keep them moist. For economic commercial growers the non-recirculating/non-recovery drip systems are most common.

The objective of this study on Asiatic hybrid lily cv. Honesty was to develop an economical way using drip hydroponic system together with the application of some nutrient solutions for obtaining lily flowers and bulblets from flower cut stem during vase life period.

\section{Material and Methods}

The investigation was conducted at the Computerized Greenhouses with Environmental Control systems where temperature ranged from 20 to $25^{\circ} \mathrm{C}$. at the Soil Research Institute (Plant Nutrition Department) in October of the two seasons of 2017 and 
2018. It was designed to study the utilization of the hydroponic technology to produce flowers and bulblets of Asiatic hybrid lily cv." Honesty" .

\section{Plant material:}

Healthy bulbs of Lilium longiflorium cv. (Honesty) were purchased from Holand in each season.

\section{Experimental design and treatments:}

A factorial experiment in a randomized complete block design was applied with two factors: (1) six solutions i.e. Hoagland No. 2 basal salt mixture, Murashige and Skoog basal salt mixture, algae extract (balanced fertilizer), Hoagland No. 2 basal salt mixture and balanced fertilizer, Hoagland No. 2 basal salt mixture and silicon beside tap -water as a control, (2) Three media components i.e. gravel, vermiculite and Hundz-soil. Each of the 6 treatments of the experiment had five replicates, each replicate contained five pots in each $(35 \mathrm{~cm})$.

\section{Preparation of the nutrient solution:}

The nutrient solutions were prepared in Soil Fertility and Plant Nutrition Dept., Soil Research Institute, and the composition of these solutions was as follows: A) Murashige and Skoog basal salt mixture (MS) ( Table, 2), B) Hoagland No. 2 basal salt mixture: ingredients include some macro and micro nutrients (Table ,3) and c) algae extract ( balance fertilizer ( Table ,4).

Table 1: Chemical analysis of tap water (meq/l).

\begin{tabular}{cccc}
\hline $\mathbf{p H}$ & 7.20 & $\mathbf{N a}^{+}$ & 2.20 \\
\hline $\mathbf{E C}(\mathbf{d} \mathbf{S} / \mathbf{m})$ & 0.40 & $\mathbf{K}^{+}$ & 0.11 \\
$\mathbf{H C O}$ & 1.24 & $\mathbf{M g}^{-3}$ & 0.92 \\
$\mathbf{S O}^{-4}$ & 1.01 & $\mathbf{C a}^{++}$ & 1.33 \\
$\mathbf{C l}^{-}$ & 2.32 & & \\
\hline
\end{tabular}

Table 2: Chemical analysis of Modified Murashige and Skoog (MS) nutrients according to Murashige and Skoog (1962).

\begin{tabular}{|c|c|}
\hline Chemical Components & Concentration ( $\mathrm{mg} / \mathrm{l}$.) \\
\hline $\mathrm{NH}_{4} \mathrm{NO}_{3}$ & 1650.00 \\
\hline $\mathrm{KNO}_{3}$ & 1900.00 \\
\hline $\mathrm{KH}_{2} \mathrm{PO}_{4}$ & 170.00 \\
\hline $\mathrm{CaCl}_{2} .2 \mathrm{H}_{2} \mathrm{O}$ & 440.00 \\
\hline $\mathrm{MgSO}_{4} .7 \mathrm{H}_{2} \mathrm{O}$ & 370.00 \\
\hline $\mathrm{H}_{3} \mathrm{BO}_{3}$ & 6.20 \\
\hline KI & 0.83 \\
\hline $\mathrm{Na}_{2} \mathrm{MoO}_{4} .2 \mathrm{H}_{2} \mathrm{O}$ & 0.25 \\
\hline $\mathrm{CaCl}_{2} .6 \mathrm{H}_{2} \mathrm{O}$ & 0.03 \\
\hline $\mathrm{MnSO}_{4} .4 \mathrm{H}_{2} \mathrm{O}$ & 22.30 \\
\hline $\mathrm{ZnSO}_{4.7 \mathrm{H}_{2} \mathrm{O}}$ & 8.60 \\
\hline $\mathrm{CuSO}_{4.5} 5 \mathrm{H}_{2} \mathrm{O}$ & 0.03 \\
\hline $\mathrm{Na}_{2}$.EDTA.2 $\mathrm{H}_{2} \mathrm{O}$ & 37.25 \\
\hline $\mathrm{FeSO}_{4} .7 \mathrm{H}_{2} \mathrm{O}$ & 27.80 \\
\hline
\end{tabular}


Table 3: Chemical analysis of Hoagland solution according to Lincoln and Zeiger (2010).

\begin{tabular}{|c|c|c|c|c|c|c|}
\hline Compound & $\begin{array}{c}\text { Molecular } \\
\text { weight } \\
\left(\mathrm{g} \mathrm{mol}^{-1}\right)\end{array}$ & $\begin{array}{c}\text { Concentration } \\
\text { of stock } \\
\text { solution } \\
(\mathrm{mM})\end{array}$ & $\begin{array}{c}\text { Concentration } \\
\text { of stock } \\
\text { solution } \\
\left(\mathrm{gL}^{-}\right)\end{array}$ & $\begin{array}{c}\text { Volume of } \\
\text { stock solution } \\
\text { per liter of } \\
\text { final solution } \\
\text { mL }\end{array}$ & Element & $\begin{array}{l}\text { Final } \\
\text { conc. of } \\
\text { element } \\
\text { (ppm) }\end{array}$ \\
\hline \multicolumn{7}{|l|}{ Macronutrients } \\
\hline $\mathrm{KNO}_{3}$ & 101.10 & 1,000 & 101.10 & 6.00 & $\mathbf{N}$ & 224 \\
\hline $\mathrm{Ca}\left(\mathrm{NO}_{3}\right)_{2-4 \mathrm{H}_{2} \mathrm{O}}$ & 236.16 & 1,000 & 236.16 & 4.00 & $\mathbf{K}$ & 235 \\
\hline $\mathrm{NH}_{4} \mathrm{H}_{2} \mathrm{PO}_{4}$ & 115.08 & 1,000 & 115.08 & 2.00 & $\begin{array}{c}\mathbf{C a} \\
\mathbf{P}\end{array}$ & $\begin{array}{c}160 \\
62\end{array}$ \\
\hline $\mathrm{MgSO}_{4}-7 \mathrm{H}_{2} \mathrm{O}$ & 246.48 & 1,000 & 246.49 & 1.00 & $\underset{\mathbf{M g}}{\mathbf{S}}$ & $\begin{array}{l}32 \\
24\end{array}$ \\
\hline \multicolumn{7}{|l|}{ Micronutrients } \\
\hline $\mathrm{KCl}$ & 74.55 & 25.00 & 246.49 & 2.00 & Cl & 1.77 \\
\hline $\mathrm{H}_{3} \mathrm{Bo3}$ & 61.83 & 12.50 & 1.86 & 2.00 & $\mathbf{B}$ & 0.27 \\
\hline $\mathrm{MnSO}_{4} \mathrm{H}_{2} \mathrm{O}$ & 169.01 & 1.00 & 0.77 & 2.00 & Mn & 0.11 \\
\hline $\mathrm{ZnSO}_{4}-7 \mathrm{H}_{2} \mathrm{O}$ & 287.54 & 1.00 & 0.17 & 2.00 & $\mathbf{Z n}$ & 0.13 \\
\hline $\mathrm{CuSO}_{4}-5 \mathrm{H}_{2} \mathrm{O}$ & 249.68 & 0.25 & 0.29 & 2.00 & $\mathbf{C u}$ & 0.03 \\
\hline $\mathrm{H}_{2} \mathrm{MoO} 4\left(85 \% \mathrm{MoO}_{3}\right)$ & 161.97 & 0.25 & 0.06 & 2.00 & Mo & 0.05 \\
\hline NaFeDTPA (10\% Fe) & 468.20 & 64.00 & 0.04 & 2.00 & $\mathbf{F e}$ & $\begin{array}{c}1.00- \\
3.00\end{array}$ \\
\hline Optional $^{\mathrm{a}} \mathrm{NiS} \mathrm{O}_{4} \cdot 6 \mathrm{H}_{2} \mathrm{O}$ & 262.86 & 0.25 & 0.07 & 1.00 & $\mathbf{N i}$ & 0.03 \\
\hline $\mathrm{Na}_{2} \mathrm{SiO}_{3}-9 \mathrm{H}_{2} \mathrm{O}$ & 284.20 & 1,00 & 284.20 & 2.00 & Si & 28.00 \\
\hline
\end{tabular}

Table 4: Chemical analysis of balanced fertilizer.

\begin{tabular}{ccc}
\hline Nutrients & Concentration (\%) \\
\hline N & $10 \%$ \\
P & $10 \%$ \\
Ka & $10 \%$ \\
Mg & $2 \%$ & $4 \%$ \\
Silica & $2 \%$ & $0 \%$ \\
Cu EDTA & $0.1 \%$ & $2 \%$ \\
Mn EDTA & $0.5 \%$ \\
\hline
\end{tabular}

Table 5: Chemical analysis of organic soil conditioner (Hundz-soil) by Hend and Awad (2015).

\begin{tabular}{|c|c|}
\hline \multicolumn{2}{|c|}{ Chemical Components } \\
\hline $\mathbf{M}^{3} / \mathbf{k g}$ & 216.00 \\
\hline Humidity \% & 3.00 \\
\hline SP \% & 278.00 \\
\hline pH (1-10) & 7.60 \\
\hline $\operatorname{EC~dSm}^{-1}(1-10)$ & 1.40 \\
\hline Total nitrogen $\%$ & 1.30 \\
\hline $\mathrm{NH}_{4} \mathrm{NO}_{3} \mu \mathrm{g} \mathrm{g}^{-1}$ & 1628.00 \\
\hline $\mathrm{NO}_{3} \mu \mathrm{g} \mathrm{g}^{-1}$ & 68.00 \\
\hline Organic matter\% & 78.20 \\
\hline Organic carbon $\%$ & 45.30 \\
\hline $\mathbf{C}: \mathbf{N}$ & $35.4: 1$ \\
\hline Total P\% & 0.08 \\
\hline Total K\% & 0.11 \\
\hline Nematode & Not found \\
\hline Seeds of grass & Not found \\
\hline parasites & Not found \\
\hline
\end{tabular}


Table 6: Analysis of some nutrient solutions in hydroponic system of Asiatic hybrid lily "Honesty" after $15,30,45$ days of planting.

\begin{tabular}{ccccccc}
\hline & \multicolumn{3}{c}{$\mathbf{p H}$} & \multicolumn{3}{c}{$\mathbf{E c}\left(\mathbf{d} \mathbf{S} / \mathbf{m}^{-\mathbf{1}}\right)$} \\
No. Treatments & $\mathbf{1 5}$ days & $\mathbf{3 0}$ days & $\mathbf{4 5}$ days & $\mathbf{1 5}$ days & $\mathbf{3 0}$ days & $\mathbf{4 5}$ days \\
\hline Water & 7.23 & 7.26 & 7.22 & 0.33 & 0.46 & 0.47 \\
B.F. & 5.73 & 5.64 & 5.55 & 1.60 & 1.88 & 1.85 \\
M.S. & 5.81 & 5.46 & 5.35 & 1.98 & 1.95 & 1.64 \\
Hoag. & 5.84 & 5.40 & 5.36 & 1.48 & 1.24 & 1.19 \\
Hoag.+Si. & 5.79 & 5.55 & 5.50 & 1.25 & 1.20 & 1.16 \\
Hoag.+B.F. & 5.81 & 5.60 & 5.00 & 1.56 & 1.55 & 1.51 \\
\hline
\end{tabular}

Table 7: Chemistry of analysis of Hundz- soil and vermiculite in hydroponic system of Asiatic hybrid lily "Honesty" after 50 days of planting.

\begin{tabular}{|c|c|c|c|c|c|c|}
\hline \multirow{2}{*}{ Treatments } & \multicolumn{3}{|c|}{ Hundz- soil } & \multicolumn{3}{|c|}{ vermiculite } \\
\hline & Total N \% & Total P \% & Total K\% & Total N\% & Total P\% & Total K\% \\
\hline Initial & 0.86 & 0.06 & 0.27 & 0.02 & 0.02 & 0.12 \\
\hline Water & 0.96 & 0.10 & 0.29 & 0.03 & 0.03 & 0.24 \\
\hline B.F. & 1.01 & 0.10 & 0.22 & 0.05 & 0.04 & 0.10 \\
\hline M.S. & 1.02 & 0.07 & 0.24 & 0.04 & 0.05 & 0.14 \\
\hline Hoag. & 1.25 & 0.15 & 0.33 & 0.07 & 0.05 & 0.07 \\
\hline Hoag.+Si. & 1.04 & 0.13 & 0.30 & 0.04 & 0.03 & 0.13 \\
\hline Hoag.+B.F. & 1.30 & 0.10 & 0.35 & 0.04 & 0.04 & 0.13 \\
\hline
\end{tabular}

Photo (1): shows Asiatic lily plants cv. "Honesty" grown in drip hydroponic system in different nutrient solutions in greenhouse. The flowers completely opened 50 days after planting with good quality in most treatments.

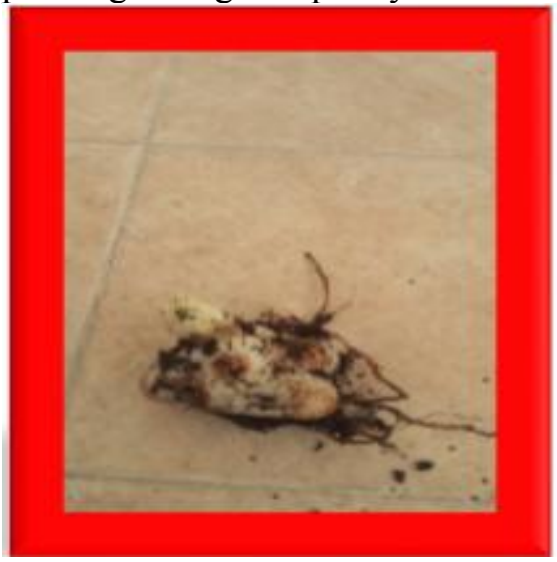

(A)

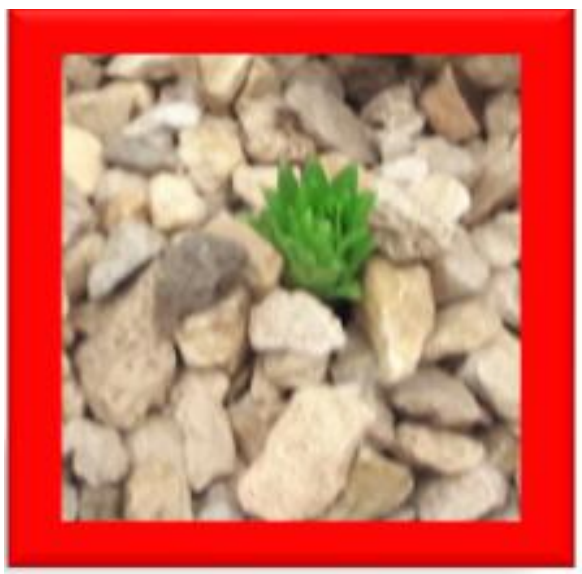

(D)

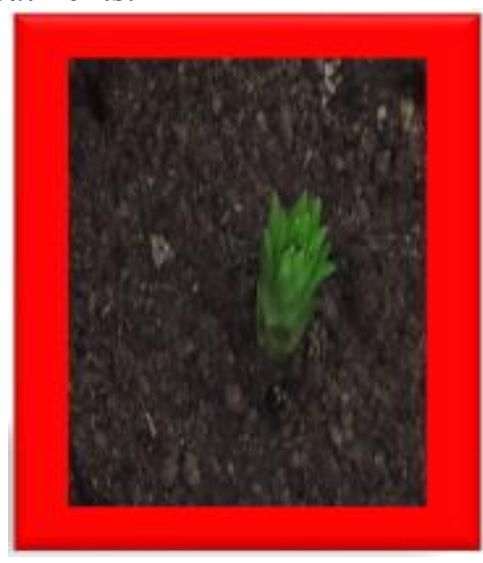

(B)

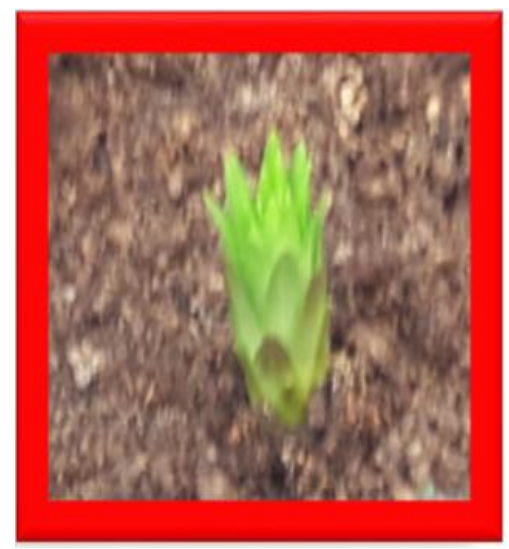

(C)

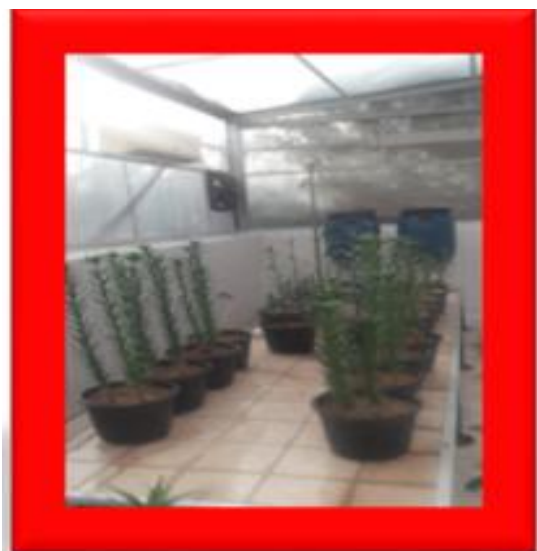

(E)

A) Bulbs after had been frozen at planting time (B) Hundz-soil (C) Vermiculite (D) Gravels (E) The growing system contained in the drip hydroponic tanks, with constant flow of nutrient solutions 
Photo (2): Asiatic lily plants cv. "Honesty" grown in drip hydroponic system in different nutrient solutions in greenhouse.

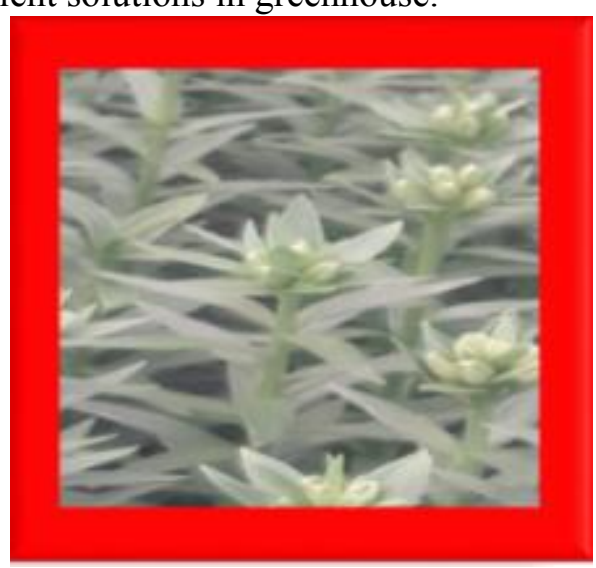

(F)

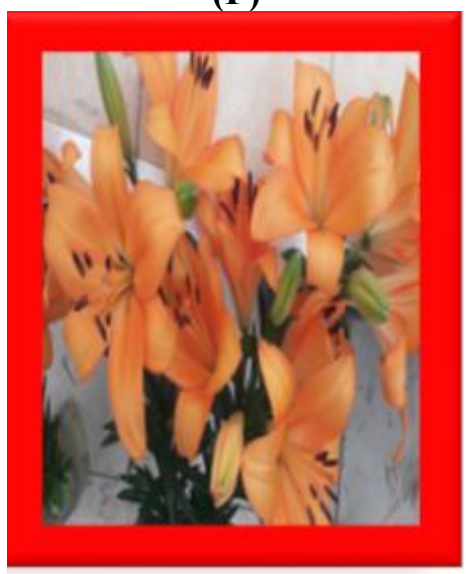

(H)

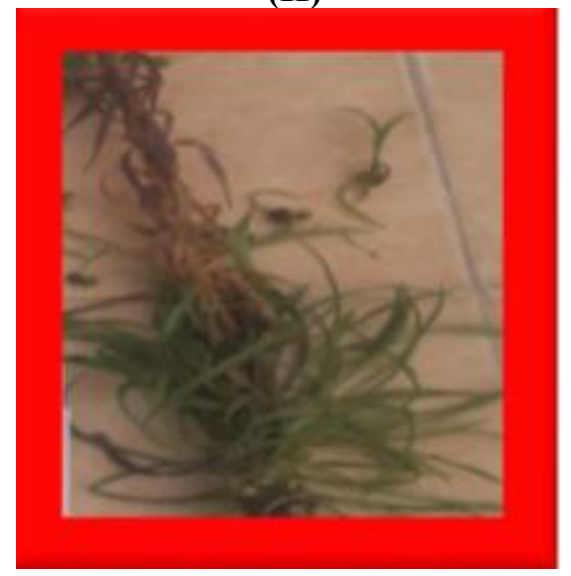

(J)

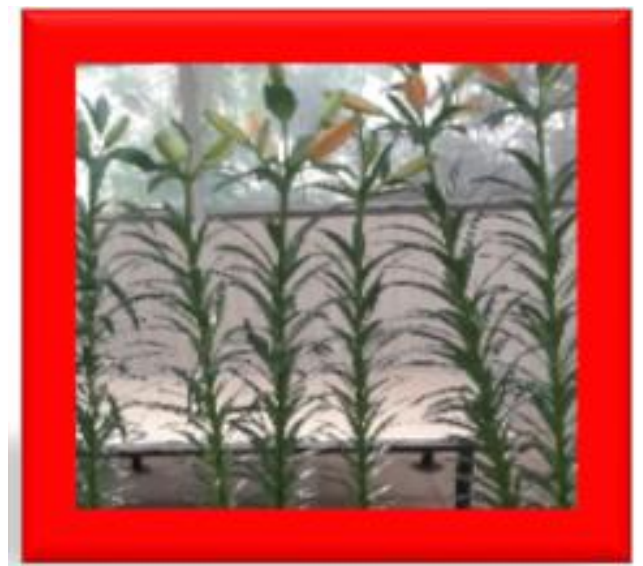

(G)

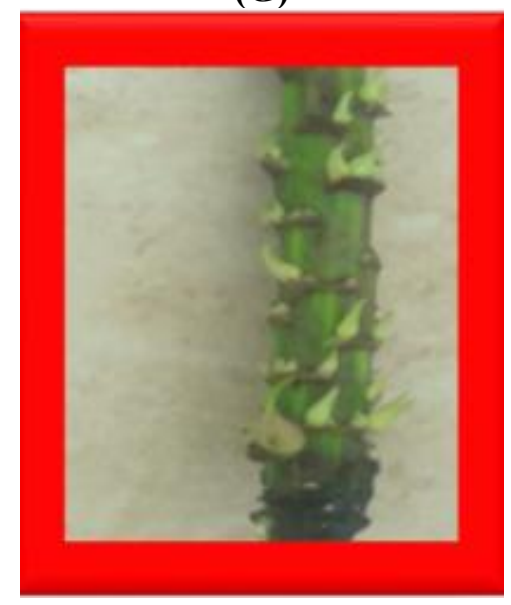

(I)

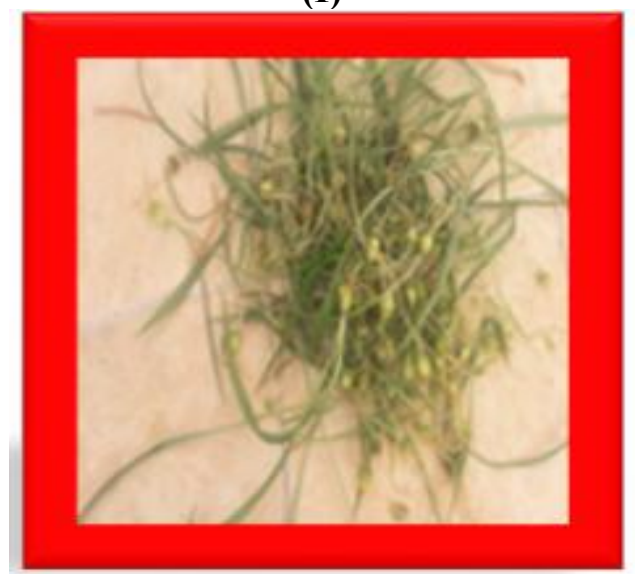

(K)

(F) Flower bud initiation (G) Developmental stage of flower buds colouring (H) The plants with full open flowers and the flowers had a good quality. (I) Flower stem cutting bulblets initiation in vase (J) More stem cutting bulblets per plant (K) Daughter bulblets containing roots. 
Photo (3): Asiatic lily plants cv. "Honesty" grown in drip hydroponic system in different nutrient solutions in greenhouse.

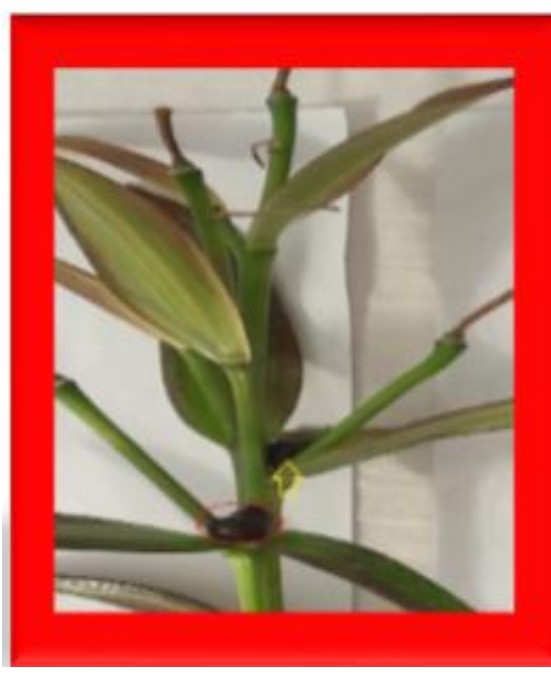

(L)

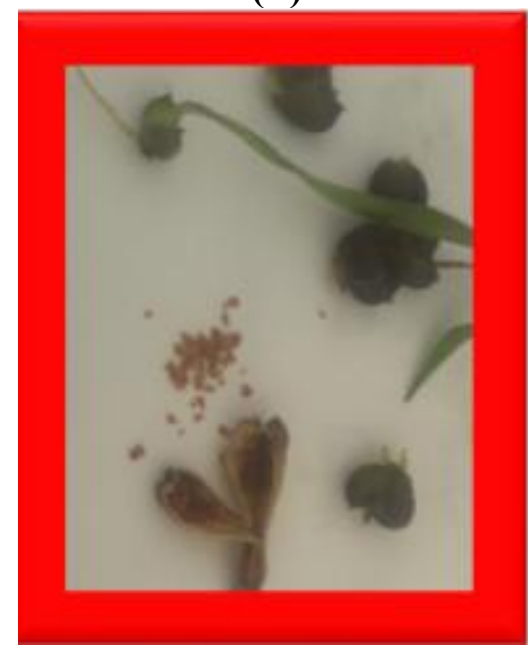

(N)

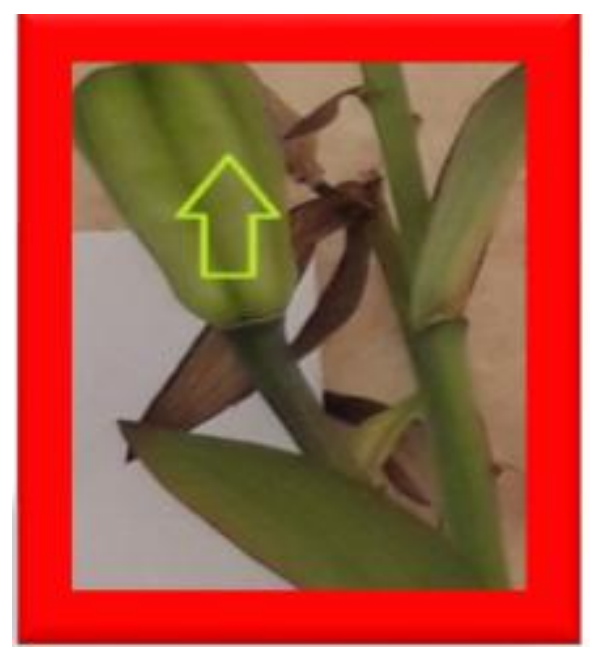

(M)

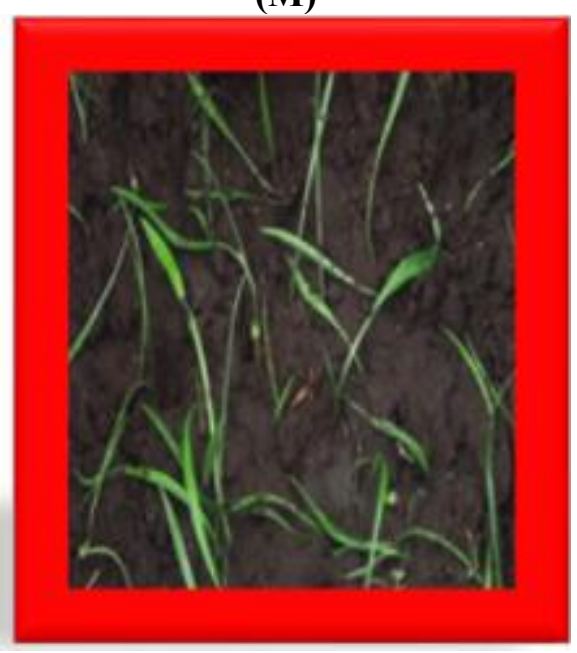

(O)

(L) Daughter bublets initiation at the top of flowering stem cuttings in vase (M) Seed capsules after vase period (N) Two types of daughter bulblets and seeds of lilium plants (O) Planting the daughter bulblets after vase periods.

\section{The following parameters were used to evaluate the tested treatments:}

1. Plant height $(\mathrm{cm})$.

2. Leaf area/plant $\left(\mathrm{cm}^{2}\right)$ : of three leaves [the fifth leaf from the top of plant] was taken to determine the mean leaf area by the apparatus MODEL 3100 AREA METER.

3. Plant fresh weight $(\mathrm{g})$.

1. Plant dry weight $(\mathrm{g})$.

2. Bulb fresh weight $(\mathrm{g})$.

3. Bulbs dry weight $(\mathrm{g})$.

4. Bulb root length $(\mathrm{cm})$.

5. Bulb diameter $(\mathrm{cm})$.

6. Number of cut stem bulblets.

7. Flower bud initiation from planting (days).

8. Flowering bud length $(\mathrm{cm})$.

9. Flowering time (days) was calculated as number of days from planting to the first inflorescence coloring. 
10.Flower stalk length $(\mathrm{cm})$.

11.Flower stem diameter $(\mathrm{cm})$.

12.Inflorescence diameter $(\mathrm{cm})$.

13. Number of inflorescences/plant.

14.Vase life/ plant (days): Three similar inflorescences from each replicate were chosen at the beginning of colouring bud stage and were cut off by a sharp knife, placed in glass jars containing tap-water directly. The tap-water was changed every 24 hours period.

15. Total chlorophyll in leaves $(\mathrm{mg} / \mathrm{g}$ fresh weight) was determined according to Moran (1982) in the fifth leaf from the top of plant.

16. Chemical analysis of leaves dried in an electric oven at $70^{\circ} \mathrm{C}$ for $48 \mathrm{hr} .0 .1 \mathrm{~g}$ of the dried sample was ground and used to determine some macro elements as follows:

17. Total $\mathrm{N}$ in leaves was determined by the modified kjeldahl UDK-130A as described by Piper (1947).

18.Total $\mathrm{P}$ in leaves was estimated calorimetrically as recommended by Troug and Meyer (1939) by spectrophotometer.

19. Total $\mathrm{K}$ in leaves was determined by flame photometer 410 (Brown and Lilliland, 1946).

\section{Statistical analysis:}

Data were tabulated and subjected to analysis of variance as a factorial experiment using MSTAT statistical software (1985). Means of treatments were compared by Duncan's Multiple Range Test at 5\% level as indicated by Snedecor and Cochran (1980).

\section{Results and Discussions:}

Effect of growing media and different nutrient solutions on vegetative growth, flowering and chemical composition of Lilium longiflorum, L.cv." Honesty".

\section{A-Effect on vegetative growth:}

These data in Tables $(8,9,10,11,12,13,14,15$, and 17) which showed that Hoagland solution with Hundz-soil as a media recorded the highest values in most treatments in the two seasons.

This may be attributed to the balance and availability of nutrients which enhances the cell metabolism resulting in better plant growth. Also, Hoagland solution provides all of the essential nutrients for plant growth and is appropriate for a wide range of plant species. In addition Hundz- soil has a natural soil conditioner that has a water-holding capacity of $300 \%$, a balanced $\mathrm{pH}$ of $6.8-7.2$ and contains essential nutrients needed for the first application, retains water longer than regular soil, so plants develop healthy root system while minimizing water loss and provides a perfect distribution of nutrients to growing plants. Our research pointed out that the plants produced more bulblets from cut flower stem during vase life period.

Results of the present experiment were in line with many researchers who revealed that the beneficial effects of Hoagland solution for vegetative growth, as observed by Michael (2002) reported that the growing medium, if any is totally inert and merely acts as a support for the plants and their root system, while the solution passes freely; Neil and Lieth (2007) explained the modeling macronutrient absorption of hydroponically-grown cut flower roses; (Wahome et al .(2010) on gladiolus and Barnes et al. (2011) on lilium longiflorum 'Nellie White' and Lilium Hybrid 'Brunello', Bever et al. (2012) noticed that the higher roots and whole plant were produced in the full Hoagland's solution; Sun el al. (2014) on three Chinese chive cultivars; Asker (2015) on Asiatic hybrid lily cv. "Blackout; Kaur et al. (2016) on tomato; Thakur (2016) on Chrysanthemum morifolium Ramat.); Safia et al. (2018) on Gladiolus (Gladiolus grandiflorus); Ranjit et al .(2018) on lettuce attained similar results.

The increase in characters of vegetative growth of plants grown on Hundz-soil and vermiculite might be attributed to better absorption the hydroponic solutions which can then be transported as free-flowing solids. Also, vermiculite has a large internal surface, high cation exchange capacity (CEC), and high negative charge on the silicate layers. 
Table 8: Effect of different nutrient solutions and different media on plant height $(\mathrm{cm})$ (of Asiatic lily (Lilium longifloum cv. Honesty) in two seasons (2017/2018 and 2018/2019).

\begin{tabular}{|c|c|c|c|c|c|c|c|c|}
\hline \multirow{3}{*}{$\begin{array}{l}\text { Treatments } \\
\text { Media }\end{array}$} & \multicolumn{8}{|c|}{ Plants height ( cm) } \\
\hline & \multicolumn{4}{|c|}{ First season $(2017 / 2018)$} & \multicolumn{4}{|c|}{ Second season $(2018 / 2019)$} \\
\hline & Gravels & Vermiculite & $\begin{array}{l}\text { Hundz- } \\
\text { soil }\end{array}$ & Mean & Gravels & Vermiculite & $\begin{array}{c}\text { Hundz- } \\
\text { soil }\end{array}$ & Mean \\
\hline Water & $69.00 \mathrm{q}$ & 80.00 o & $77.00 \mathrm{p}$ & 75.33 F & $73.11 \mathrm{p}$ & 81.65 o & $82.13 \mathrm{n}$ & $78.96 \mathrm{~F}$ \\
\hline B.F. & $91.00 \mathrm{k}$ & $93.33 \mathrm{i}$ & $100.00 \mathrm{e}$ & 94.78 D & 92.021 & $97.36 \mathrm{~h}$ & $105.12 \mathrm{~d}$ & 98.17 D \\
\hline M.S. & $86.00 \mathrm{~m}$ & $97.33 \mathrm{f}$ & $104.00 \mathrm{c}$ & $95.67 \mathrm{C}$ & $89.00 \mathrm{~m}$ & $98.30 \mathrm{f}$ & $103.10 \mathrm{e}$ & $96.80 \mathrm{E}$ \\
\hline Hoag. & $93.00 \mathrm{j}$ & $95.00 \mathrm{~h}$ & $110.00 \mathrm{a}$ & $99.33 \mathrm{~A}$ & $96.01 \mathrm{j}$ & $98.02 \mathrm{~g}$ & $112.98 \mathrm{a}$ & $102.34 \mathrm{~A}$ \\
\hline Hoag. +Si. & $91.00 \mathrm{k}$ & 90.001 & $109.00 \mathrm{~b}$ & 96.67 B & $96.01 \mathrm{j}$ & $97.02 \mathrm{i}$ & $111.82 \mathrm{~b}$ & $101.62 \mathrm{~B}$ \\
\hline Hoag. + BF. & $83.33 \mathrm{n}$ & $96.00 \mathrm{~g}$ & $101.00 \mathrm{~d}$ & $93.44 \mathrm{E}$ & $93.31 \mathrm{k}$ & $98.02 \mathrm{~g}$ & $111.20 \mathrm{c}$ & $100.85 \mathrm{C}$ \\
\hline Mean & $85.56 \mathrm{C}$ & $91.89 \mathrm{~B}$ & $100.17 \mathrm{~A}$ & & 89.91C c & $95.06 \mathrm{~B}$ & $104.39 \mathrm{~A}$ & \\
\hline
\end{tabular}

Means followed by the same letters in a column or raw do not differ significantly according to Duncan's New Multiple Range test at $\mathrm{P}=0.05$.

Table 9: Effect of different nutrient solutions and different media on leaf area/plant $\left(\mathrm{cm}^{2}\right)$ of Asiatic lily (Lilium longifloum cv. Honesty in two seasons (2017/2018 and 2018/2019).

\begin{tabular}{|c|c|c|c|c|c|c|c|c|}
\hline \multirow{3}{*}{$\begin{array}{l}\text { Treatments } \\
\text { Media } \\
\text { Solutions }\end{array}$} & \multicolumn{8}{|c|}{ Leaf area/plant $\left(\mathrm{cm}^{2}\right)$} \\
\hline & \multicolumn{4}{|c|}{ First season $(2017 / 2018)$} & \multicolumn{4}{|c|}{ Second season $(2018 / 2019)$} \\
\hline & Gravels & Vermiculite & $\begin{array}{c}\text { Hundz- } \\
\text { soil }\end{array}$ & Mean & Gravels & Vermiculite & $\begin{array}{c}\text { Hundz- } \\
\text { soil }\end{array}$ & Mean \\
\hline Water & $7.08 \mathrm{p}$ & $7.80 \mathrm{n}$ & 8.861 & $7.91 \mathrm{~F}$ & $7.12 \mathrm{p}$ & 7.56 o & $8.57 \mathrm{~m}$ & $7.75 \mathrm{~F}$ \\
\hline B.F. & $9.91 \mathrm{~g}$ & $9.43 \mathrm{j}$ & $10.96 \mathrm{c}$ & $10.0 \mathrm{~B}$ & 9.021 & $9.99 \mathrm{~h}$ & $10.61 \mathrm{e}$ & $9.87 \mathrm{~B}$ \\
\hline M.S. & $7.56 \mathrm{o}$ & $10.87 \mathrm{~d}$ & $11.23 \mathrm{~b}$ & $9.89 \mathrm{D}$ & $7.03 \mathrm{q}$ & $10.98 \mathrm{c}$ & $10.91 \mathrm{~d}$ & $9.64 \mathrm{D}$ \\
\hline Hoag. & $9.78 \mathrm{~h}$ & $11.23 b$ & $11.46 \mathrm{a}$ & $10.82 \mathrm{~A}$ & $9.74 \mathrm{j}$ & $11.02 \mathrm{~b}$ & $11.62 \mathrm{a}$ & $10.79 \mathrm{~A}$ \\
\hline Hoag. +Si. & $9.70 \mathrm{i}$ & $9.79 \mathrm{~h}$ & $10.46 \mathrm{e}$ & $9.99 \mathrm{C}$ & $9.87 \mathrm{i}$ & 9.011 & $10.16 \mathrm{~g}$ & $9.68 \mathrm{C}$ \\
\hline Hoag. + BF. & $8.38 \mathrm{~m}$ & $9.24 \mathrm{k}$ & $10.19 \mathrm{f}$ & $9.27 \mathrm{E}$ & $8.47 \mathrm{n}$ & $9.11 \mathrm{k}$ & $10.23 \mathrm{f}$ & $9.27 \mathrm{E}$ \\
\hline Mean & $8.73 \mathrm{C}$ & $9.73 \mathrm{~B}$ & $10.53 \mathrm{~A}$ & & $8.54 \mathrm{C}$ & $9.87 \mathrm{~B}$ & $10.09 \mathrm{~A}$ & \\
\hline
\end{tabular}

Means followed by the same letters in a column or raw do not differ significantly according to Duncan's New Multiple Range test at $\mathrm{P}=0.05$.

Table 10: Effect of different solutions and different media on plant fresh weight (g) of Asiatic lily (Lilium longifloum cv. Honesty) in two seasons (2017/2018 and 2018/2019).

\begin{tabular}{|c|c|c|c|c|c|c|c|c|}
\hline \multirow{3}{*}{$\begin{array}{l}\text { Treatments } \\
\text { Media } \\
\text { Solutions }\end{array}$} & \multicolumn{8}{|c|}{ Plant fresh weight /plant( g) } \\
\hline & \multicolumn{4}{|c|}{ First season $(2017 / 2018)$} & \multicolumn{4}{|c|}{ Second season $(2018 / 2019)$} \\
\hline & Gravels & Vermiculite & $\begin{array}{c}\text { Hundz- } \\
\text { soil }\end{array}$ & Mean & Gravels & Vermiculite & $\begin{array}{c}\text { Hundz- } \\
\text { soil }\end{array}$ & Mean \\
\hline Water & $67.73 \mathrm{r}$ & $90.80 \mathrm{p}$ & $106.35 \mathrm{k}$ & 88.29 F & $68.73 \mathrm{r}$ & $93.73 \mathrm{o}$ & 106.351 & $89.60 \mathrm{~F}$ \\
\hline B.F. & $76.13 \mathrm{q}$ & $107.00 \mathrm{i}$ & $119.92 \mathrm{~g}$ & $101.02 \mathrm{E}$ & $75.15 \mathrm{q}$ & $110.00 \mathrm{j}$ & $121.33 \mathrm{f}$ & $102.16 \mathrm{E}$ \\
\hline M.S. & $91.67 \mathrm{o}$ & $122.67 \mathrm{c}$ & $120.16 \mathrm{f}$ & 111.50 B & $93.56 \mathrm{p}$ & $120.99 \mathrm{~g}$ & $125.19 \mathrm{~d}$ & 113.24 D \\
\hline Hoag. & $100.24 \mathrm{~m}$ & $133.72 \mathrm{~b}$ & $141.76 \mathrm{a}$ & $125.24 \mathrm{~A}$ & $102.24 \mathrm{~m}$ & $135.17 \mathrm{~b}$ & $140.79 \mathrm{a}$ & $126.07 \mathrm{~A}$ \\
\hline Hoag. + + Si. & $99.00 \mathrm{n}$ & $108.33 \mathrm{~h}$ & $121.54 \mathrm{e}$ & 109.18 D & $98.90 \mathrm{n}$ & $110.30 \mathrm{i}$ & $132.50 \mathrm{c}$ & $113.90 \mathrm{C}$ \\
\hline Hoag. + BF. & 101.391 & $106.66 \mathrm{j}$ & $121.96 \mathrm{~d}$ & $110.00 \mathrm{C}$ & $108.39 \mathrm{k}$ & $119.31 \mathrm{~h}$ & $121.96 \mathrm{e}$ & $116.56 \mathrm{~B}$ \\
\hline Mean & $89.36 \mathrm{C}$ & 111.53B & $121.95 \mathrm{~A}$ & & 91.16C & 114.92 B & 124.69A & \\
\hline
\end{tabular}

Means followed by the same letters in a column or raw do not differ significantly according to Duncan's New Multiple Range test at $\mathrm{P}=0.05$.

Table 11: Effect of different nutrient solutions and different media on plant dry weight $(\mathrm{g})$ of Asiatic lily (Lilium longifloum cv. Honesty in two seasons (2017/2018 and 2018/2019).

\begin{tabular}{|c|c|c|c|c|c|c|c|c|}
\hline \multirow{3}{*}{$\begin{array}{l}\text { Treatments } \\
\text { Media } \\
\text { Solutions }\end{array}$} & \multicolumn{8}{|c|}{ Plant dry weight (g) } \\
\hline & \multicolumn{4}{|c|}{ First season $(2017 / 2018)$} & \multicolumn{4}{|c|}{ Second season $(2018 / 2019)$} \\
\hline & Gravels & Vermiculite & $\begin{array}{c}\text { Hundz- } \\
\text { soil }\end{array}$ & Mean & Gravels & Vermiculite & $\begin{array}{c}\text { Hundz- } \\
\text { soil }\end{array}$ & Mean \\
\hline Water & $14.27 \mathrm{r}$ & $19.16 \mathrm{p}$ & $22.57 \mathrm{k}$ & $18.67 \mathrm{~F}$ & $15.16 \mathrm{p}$ & $19.90 \mathrm{n}$ & $23.87 \mathrm{i}$ & $19.64 \mathrm{~F}$ \\
\hline B.F. & $16.30 \mathrm{q}$ & $22.70 \mathrm{i}$ & $25.33 \mathrm{~g}$ & $21.44 \mathrm{E}$ & $17.30 \mathrm{o}$ & $23.70 \mathrm{j}$ & $25.76 \mathrm{e}$ & $22.25 \mathrm{E}$ \\
\hline M.S. & $19.37 \mathrm{o}$ & $25.98 \mathrm{c}$ & $25.54 \mathrm{f}$ & 23.63 B & $19.90 \mathrm{n}$ & $25.55 \mathrm{f}$ & $26.61 \mathrm{~d}$ & 24.02 D \\
\hline Hoag. & $21.29 \mathrm{n}$ & $28.38 \mathrm{~b}$ & $30.02 \mathrm{a}$ & $26.54 \mathrm{~A}$ & 22.791 & $28.75 \mathrm{c}$ & $29.81 \mathrm{a}$ & $27.11 \mathrm{~A}$ \\
\hline Hoag. +Si. & 21.611 & $22.99 \mathrm{~h}$ & $25.79 \mathrm{~d}$ & $23.47 \mathrm{C}$ & $22.86 \mathrm{k}$ & $24.32 \mathrm{~h}$ & $28.79 \mathrm{~b}$ & $25.30 \mathrm{C}$ \\
\hline Hoag. + BF. & $21.50 \mathrm{~m}$ & $22.65 \mathrm{j}$ & $25.74 \mathrm{e}$ & $23.30 \mathrm{D}$ & $22.50 \mathrm{~m}$ & $24.85 \mathrm{~g}$ & $28.74 \mathrm{c}$ & 25.37 B \\
\hline Mean & $19.06 \mathrm{C}$ & $23.63 \mathrm{~B}$ & $25.83 \mathrm{~A}$ & & $20.09 \mathrm{C}$ & $24.50 B$ & $27.26 \mathrm{~A}$ & \\
\hline
\end{tabular}

Means followed by the same letters in a column or raw do not differ significantly according to Duncan's New Multiple Range test at $\mathrm{P}=0.05$. 
Table 12: Effect of different solutions and different media on bulbs fresh weight (g) of Asiatic lily (Lilium longifloum cv. Honesty) in two seasons (2017/2018 and 2018/2019).

\begin{tabular}{|c|c|c|c|c|c|c|c|c|}
\hline \multirow{3}{*}{$\begin{array}{l}\begin{array}{l}\text { Treatments } \\
\text { Media }\end{array} \\
\text { Solutions } \\
\end{array}$} & \multicolumn{8}{|c|}{ Bulbs fresh weight (g) } \\
\hline & \multicolumn{4}{|c|}{ First season $(2017 / 2018)$} & \multicolumn{4}{|c|}{ Second season $(2018 / 2019)$} \\
\hline & Gravels & Vermiculite & $\begin{array}{c}\text { Hundz- } \\
\text { soil }\end{array}$ & Mean & Gravels & Vermiculite & $\begin{array}{c}\text { Hundz- } \\
\text { soil }\end{array}$ & Mean \\
\hline Water & $24.00 \mathrm{q}$ & $21.33 \mathrm{r}$ & 29.47 o & $24.93 \mathrm{~F}$ & $29.46 \mathrm{p}$ & $26.33 \mathrm{q}$ & $31.57 \mathrm{o}$ & $29.12 \mathrm{~F}$ \\
\hline B.F. & $27.08 \mathrm{p}$ & $39.00 \mathrm{f}$ & $35.54 \mathrm{~h}$ & 33.87 E & $32.08 \mathrm{n}$ & $37.26 \mathrm{~g}$ & $39.54 \mathrm{e}$ & $36.29 \mathrm{E}$ \\
\hline M.S. & $32.33 \mathrm{k}$ & $35.67 \mathrm{~g}$ & $41.67 \mathrm{~d}$ & $36.56 \mathrm{~B}$ & $34.36 \mathrm{j}$ & $37.61 \mathrm{f}$ & $43.65 \mathrm{~d}$ & 38.54 B \\
\hline Hoag. & $33.12 \mathrm{i}$ & $44.00 \mathrm{c}$ & $49.33 \mathrm{a}$ & 42.15 A & $35.12 \mathrm{i}$ & $46.00 \mathrm{~b}$ & $50.96 \mathrm{a}$ & $44.03 \mathrm{~A}$ \\
\hline Hoag. +Si. & 31.151 & $33.00 \mathrm{j}$ & $44.05 \mathrm{~b}$ & 36.07 C & $33.15 \mathrm{~m}$ & $36.00 \mathrm{~h}$ & $45.99 \mathrm{~b}$ & $38.38 \mathrm{C}$ \\
\hline Hoag. + BF. & $31.00 \mathrm{~m}$ & $30.96 \mathrm{n}$ & $40.05 \mathrm{e}$ & 34.00 D & $34.01 \mathrm{k}$ & 33.301 & $43.90 \mathrm{c}$ & $37.07 \mathrm{D}$ \\
\hline Mean & $29.78 \mathrm{C}$ & $33.99 \mathrm{~B}$ & $40.02 \mathrm{~A}$ & & $33.03 \mathrm{C}$ & 36.08 B & $42.60 \mathrm{~A}$ & \\
\hline
\end{tabular}

Means followed by the same letters in a column or raw do not differ significantly according to Duncan's New Multiple Range test at $\mathrm{P}=0.05$.

Table 13: Effect of different nutrient solutions and different media on bulbs dry weight (g) of Asiatic lily (Lilium longifloum cv. Honesty) in two seasons (2017/2018 and 2018/2019).

\begin{tabular}{|c|c|c|c|c|c|c|c|c|}
\hline \multirow{3}{*}{$\begin{array}{r}\text { Treatments } \\
\text { Media } \\
\text { Solutions }\end{array}$} & \multicolumn{8}{|c|}{ Bulbs dry weight (g) } \\
\hline & \multicolumn{4}{|c|}{ First season (2017/2018) } & \multicolumn{4}{|c|}{ Second season $(2018 / 2019)$} \\
\hline & Gravels & Vermiculite & $\begin{array}{c}\text { Hundz- } \\
\text { soil }\end{array}$ & Mean & Gravels & Vermiculite & $\begin{array}{c}\text { Hundz- } \\
\text { soil }\end{array}$ & Mean \\
\hline Water & $4.99 \mathrm{p}$ & 5.560 & $6.39 \mathrm{~m}$ & $5.65 \mathrm{~F}$ & $5.72 \mathrm{q}$ & $6.38 \mathrm{p}$ & 6.82 o & $6.31 \mathrm{~F}$ \\
\hline B.F. & $5.94 \mathrm{n}$ & $8.60 \mathrm{~d}$ & $7.07 \mathrm{~h}$ & $7.20 \mathrm{E}$ & $7.04 \mathrm{n}$ & $8.01 \mathrm{~h}$ & $8.59 \mathrm{f}$ & $7.88 \mathrm{E}$ \\
\hline M.S. & $7.04 \mathrm{i}$ & $7.76 \mathrm{f}$ & $9.03 \mathrm{c}$ & $7.94 \mathrm{~B}$ & 7.491 & $8.28 \mathrm{~g}$ & $9.07 \mathrm{~d}$ & $8.28 \mathrm{C}$ \\
\hline Hoag. & $7.27 \mathrm{~g}$ & $9.69 \mathrm{~b}$ & $10.72 \mathrm{a}$ & $9.23 \mathrm{~A}$ & $7.71 \mathrm{k}$ & $10.13 \mathrm{~b}$ & $10.46 \mathrm{a}$ & $9.44 \mathrm{~A}$ \\
\hline Hoag. +Si. & $6.83 \mathrm{k}$ & $7.26 \mathrm{~g}$ & $9.69 \mathrm{~b}$ & $7.92 \mathrm{C}$ & $7.26 \mathrm{~m}$ & $7.92 \mathrm{i}$ & $10.10 \mathrm{c}$ & 8.44 B \\
\hline Hoag.+ BF. & $6.85 \mathrm{j}$ & 6.811 & $8.09 \mathrm{e}$ & $7.25 \mathrm{D}$ & $7.27 \mathrm{~m}$ & $7.87 \mathrm{j}$ & $9.05 \mathrm{e}$ & $8.06 \mathrm{D}$ \\
\hline Mean & $6.49 \mathrm{C}$ & $7.61 \mathrm{~B}$ & $8.50 \mathrm{~A}$ & & $7.08 \mathrm{C}$ & $8.10 \mathrm{~B}$ & $9.01 \mathrm{~A}$ & \\
\hline
\end{tabular}

Means followed by the same letters in a column or raw do not differ significantly according to Duncan's New Multiple Range test at $\mathrm{P}=0.05$.

Table 14: Effect of different nutrient solutions and different media on bulbs root length (cm) of Asiatic lily (Lilium longifloum cv. Honesty) in two seasons (2017/2018 and 2018/2019).

\begin{tabular}{|c|c|c|c|c|c|c|c|c|}
\hline \multirow{3}{*}{$\begin{array}{l}\text { Treatments } \\
\text { Media }\end{array}$} & \multicolumn{8}{|c|}{ Bulbs root length ( $\mathrm{cm}$ ) } \\
\hline & \multicolumn{4}{|c|}{ First season $(2017 / 2018)$} & \multicolumn{4}{|c|}{ Second season $(2018 / 2019)$} \\
\hline & Gravels & Vermiculite & $\begin{array}{c}\text { Hundz- } \\
\text { soil }\end{array}$ & Mean & Gravels & Vermiculite & $\begin{array}{l}\text { Hundz- } \\
\text { soil }\end{array}$ & Mean \\
\hline Water & $9.33 \mathrm{p}$ & $12.67 \mathrm{n}$ & $10.67 \mathrm{o}$ & $10.89 \mathrm{~F}$ & $6.11 \mathrm{p}$ & $11.69 \mathrm{o}$ & $12.37 \mathrm{n}$ & $10.06 \mathrm{~F}$ \\
\hline B.F. & $13.67 \mathrm{k}$ & $13.67 \mathrm{k}$ & 13.611 & $13.65 \mathrm{E}$ & $12.57 \mathrm{~m}$ & 13.561 & $14.12 \mathrm{k}$ & $13.42 \mathrm{E}$ \\
\hline M.S. & $14.67 \mathrm{j}$ & $17.67 \mathrm{e}$ & $16.62 \mathrm{f}$ & $16.32 \mathrm{C}$ & $15.74 \mathrm{j}$ & $16.67 \mathrm{~h}$ & $17.65 \mathrm{f}$ & 16.69 D \\
\hline Hoag. & $16.33 \mathrm{~g}$ & $18.00 \mathrm{~d}$ & $19.65 \mathrm{~b}$ & $17.99 \mathrm{~A}$ & $16.97 \mathrm{~g}$ & $19.52 \mathrm{c}$ & $20.62 \mathrm{a}$ & $19.04 \mathrm{~A}$ \\
\hline Hoag. +Si. & $13.33 \mathrm{~m}$ & $15.33 \mathrm{i}$ & $18.67 \mathrm{c}$ & $15.78 \mathrm{D}$ & $16.38 \mathrm{i}$ & $19.30 \mathrm{~d}$ & $20.62 \mathrm{a}$ & $18.77 \mathrm{~B}$ \\
\hline Hoag. + BF. & $15.33 \mathrm{i}$ & $15.56 \mathrm{~h}$ & $19.67 \mathrm{a}$ & $17.00 \mathrm{~B}$ & $16.37 \mathrm{i}$ & $18.15 \mathrm{e}$ & $19.98 \mathrm{~b}$ & 18.16 C \\
\hline Mean & $13.78 \mathrm{C}$ & 15.56 & $16.48 \mathrm{~A}$ & & $13.42 \mathrm{C}$ & 14.02 B & $16.48 \mathrm{~A}$ & \\
\hline
\end{tabular}

Means followed by the same letters in a column or raw do not differ significant according to Duncan's New Multiple Range test at $\mathrm{P}=0.05$.

Table 15: Effect of different nutrient solutions and different media on bulbs diameter (cm) of Asiatic lily (Lilium longifloum cv. Honesty) in two seasons (2017/2018 and 2018/2019).

\begin{tabular}{|c|c|c|c|c|c|c|c|c|}
\hline \multirow{3}{*}{$\begin{array}{c}\text { Treatments } \\
\text { Media } \\
\text { Solutions }\end{array}$} & \multicolumn{8}{|c|}{ Bulbs diameter ( $\mathbf{c m})$} \\
\hline & \multicolumn{4}{|c|}{ First season $(2017 / 2018)$} & \multicolumn{4}{|c|}{ Second season $(2018 / 2019)$} \\
\hline & Gravels & Vermiculite & $\begin{array}{l}\text { Hundz- } \\
\text { soil }\end{array}$ & Mean & Gravels & Vermiculite & $\begin{array}{l}\text { Hundz- } \\
\text { soil }\end{array}$ & Mean \\
\hline Water & $3.33 \mathrm{n}$ & $3.57 \mathrm{j}$ & $3.10 \mathrm{o}$ & $3.33 \mathrm{E}$ & $2.93 \mathrm{q}$ & $3.68 \mathrm{k}$ & $3.56 \mathrm{~m}$ & $3.39 \mathrm{~F}$ \\
\hline B.F. & $3.63 \mathrm{i}$ & $3.67 \mathrm{~h}$ & $3.40 \mathrm{~m}$ & $3.57 \mathrm{D}$ & 3.601 & $3.74 \mathrm{j}$ & $3.12 \mathrm{p}$ & $3.49 \mathrm{E}$ \\
\hline M.S. & 3.471 & $3.70 \mathrm{~g}$ & $4.17 \mathrm{~b}$ & $3.78 \mathrm{~B}$ & $3.36 \mathrm{n}$ & $3.27 \mathrm{o}$ & $4.39 \mathrm{~d}$ & 3.67 D \\
\hline Hoag. & $3.87 \mathrm{e}$ & $4.20 \mathrm{a}$ & $4.07 \mathrm{c}$ & $4.04 \mathrm{~A}$ & $3.92 \mathrm{~h}$ & $4.35 \mathrm{e}$ & $4.56 \mathrm{a}$ & $4.28 \mathrm{~A}$ \\
\hline Hoag. +Si. & $3.40 \mathrm{~m}$ & $3.83 \mathrm{f}$ & $4.02 \mathrm{~d}$ & $3.75 \mathrm{C}$ & $3.13 \mathrm{p}$ & $4.11 \mathrm{f}$ & $4.48 \mathrm{~b}$ & $3.90 \mathrm{C}$ \\
\hline Hoag. + BF. & $4.03 \mathrm{~d}$ & $3.53 \mathrm{k}$ & $3.87 \mathrm{e}$ & $3.81 \mathrm{~B}$ & $3.94 \mathrm{~g}$ & $3.88 \mathrm{i}$ & $4.42 \mathrm{c}$ & $4.08 \mathrm{~B}$ \\
\hline Mean & $3.62 \mathrm{C}$ & $3.75 \mathrm{~B}$ & $3.89 \mathrm{~A}$ & & $3.48 \mathrm{C}$ & $3.84 \mathrm{~B}$ & 4.09A & \\
\hline
\end{tabular}

Means followed by the same letters in a column or raw do not differ significantly according to Duncan's New Multiple Range test at $\mathrm{P}=0.05$. 
Table 16: Effect of different nutrient solutions and different media on number of cut stem bulblets of Asiatic lily (lilium longifloum cv. Honesty) in two seasons (2017/2018 and 2018/2019).

\begin{tabular}{|c|c|c|c|c|c|c|c|c|}
\hline \multirow{3}{*}{$\begin{array}{l}\text { Treatments } \\
\text { Media } \\
\text { Solutions }\end{array}$} & \multicolumn{8}{|c|}{ Number of cut stem bulblets } \\
\hline & \multicolumn{4}{|c|}{ First season $(2017 / 2018)$} & \multicolumn{4}{|c|}{ Second season $(2018 / 2019)$} \\
\hline & Gravels & Vermiculite & $\begin{array}{c}\text { Hundz- } \\
\text { soil }\end{array}$ & Mean & Gravels & Vermiculite & $\begin{array}{l}\text { Hundz- } \\
\text { soil }\end{array}$ & Mean \\
\hline Water & $43.10 \mathrm{r}$ & $50.67 \mathrm{q}$ & 54.00 o & $49.26 \mathrm{~F}$ & $43.10 \mathrm{r}$ & $49.15 \mathrm{q}$ & $51.24 \mathrm{o}$ & $47.83 \mathrm{E}$ \\
\hline B.F. & $51.02 \mathrm{p}$ & $60.33 n$ & $69.67 \mathrm{~h}$ & $60.34 \mathrm{E}$ & $50.00 \mathrm{p}$ & $61.34 \mathrm{n}$ & $70.89 \mathrm{i}$ & 60.75 D \\
\hline M.S. & 65.671 & $74.00 \mathrm{f}$ & $78.00 \mathrm{~b}$ & $72.56 \mathrm{~B}$ & $66.67 \mathrm{~m}$ & $76.02 \mathrm{~g}$ & $79.90 \mathrm{~b}$ & $75.37 \mathrm{~A}$ \\
\hline Hoag. & $67.00 \mathrm{j}$ & $75.00 \mathrm{e}$ & $79.00 \mathrm{a}$ & 73.67 A & $68.10 \mathrm{j}$ & $78.00 \mathrm{e}$ & $80.02 \mathrm{a}$ & $75.37 \mathrm{~A}$ \\
\hline Hoag. +Si. & $61.67 \mathrm{~m}$ & $71.33 \mathrm{~g}$ & $76.33 \mathrm{~d}$ & 69.78 D & 67.651 & $76.40 \mathrm{f}$ & $78.36 \mathrm{~d}$ & 74.14 B \\
\hline Hoag. + BF. & $66.01 \mathrm{k}$ & $69.00 \mathrm{i}$ & $77.00 \mathrm{c}$ & $70.67 \mathrm{C}$ & $68.05 \mathrm{k}$ & $75.00 \mathrm{~h}$ & $79.01 \mathrm{c}$ & $74.02 \mathrm{C}$ \\
\hline Mean & 59.08 C & 66.72 B & $72.33 \mathrm{~A}$ & & $60.60 \mathrm{C}$ & 69.32 B & 73.23A & \\
\hline
\end{tabular}

Table 17: Effect of the best media, solution and their interaction on some vegetative growth of Asiatic lily (Lilium longifloum cv. Honesty) in two seasons (2017/2018 and 2018/2019).

\begin{tabular}{|c|c|c|c|c|c|c|c|c|c|c|}
\hline \multirow[t]{2}{*}{ Treatments } & \multicolumn{2}{|c|}{$\begin{array}{l}\text { Plant height } \\
\text { (cm) }\end{array}$} & \multicolumn{2}{|c|}{$\begin{array}{l}\text { Leaf area/plant } \\
\qquad\left(\mathrm{cm}^{2}\right)\end{array}$} & \multicolumn{2}{|c|}{$\begin{array}{c}\text { Plant fresh weight } \\
\text { (g.) }\end{array}$} & \multicolumn{2}{|c|}{$\begin{array}{l}\text { Plant dry } \\
\text { weight } \\
\text { (g.) }\end{array}$} & \multicolumn{2}{|c|}{$\begin{array}{c}\text { Bulb fresh w. } \\
\text { (g) }\end{array}$} \\
\hline & 1st. & $2 n d$. & 1 st. & 2nd. & 1st. & 2nd. & 1st. & $2 n d$. & 1st. & $2 n d$. \\
\hline Water (control) & 75.33 & 78.96 & 7.91 & 7.75 & 88.29 & 89.60 & 18.67 & 19.64 & 24.93 & 29.12 \\
\hline Hoag. solution & 99.33 & 102.34 & 10.82 & 10.79 & 125.24 & 126.07 & 26.54 & 27.11 & 42.15 & 44.03 \\
\hline Gravels & 85.56 & 89.91 & 8.73 & 8.54 & 89.36 & 91.16 & 19.06 & 20.09 & 29.78 & 33.03 \\
\hline Hundz-soil & 100.17 & 104.39 & 10.53 & 10.09 & 121.95 & 124.69 & 25.83 & 27.26 & 40.02 & 42.60 \\
\hline $\begin{array}{l}\text { Interaction } \\
\text { (Hoag.+Hundz } \\
\text { soil) }\end{array}$ & 110.00 & 112.98 & 11.46 & 11.62 & 141.76 & 140.79 & 30.02 & 29.81 & 49.33 & 50.96 \\
\hline
\end{tabular}

Table 17: Continued

\begin{tabular}{|c|c|c|c|c|c|c|c|c|}
\hline \multirow[t]{2}{*}{ Treatments } & \multicolumn{2}{|c|}{$\begin{array}{c}\text { Bulb dry w. } \\
\text { (g.) }\end{array}$} & \multicolumn{2}{|c|}{$\begin{array}{l}\text { Bulb root } \\
\text { length }(\mathrm{cm})\end{array}$} & \multicolumn{2}{|c|}{$\begin{array}{l}\text { Bulb diameter } \\
(\mathrm{cm})\end{array}$} & \multicolumn{2}{|c|}{$\begin{array}{l}\text { N. of cut stem } \\
\text { bulblets }\end{array}$} \\
\hline & 1st. & 2nd. & 1st. & 2nd. & 1st. & 2nd. & 1st. & 2nd. \\
\hline Water (control) & 5.65 & 6.31 & 10.89 & 10.06 & 3.33 & 3.39 & 49.26 & 47.83 \\
\hline Hoag. solution & 9.23 & 9.44 & 17.99 & 19.04 & 4.04 & 4.28 & 73.67 & 75.37 \\
\hline Gravels & 6.49 & 7.08 & 13.78 & 13.42 & 3.62 & 3.48 & 59.08 & 60.60 \\
\hline Hundz-soil & 8.50 & 9.01 & 16.48 & 16.48 & 3.89 & 4.09 & 72.33 & 73.23 \\
\hline $\begin{array}{l}\text { Interaction } \\
\text { (Hoag.+Hundz soil) }\end{array}$ & 10.72 & 10.46 & 19.65 & 20.62 & 4.07 & 4.56 & 79.00 & 80.02 \\
\hline
\end{tabular}

1st.=First season, 2nd. $=$ Second season

\section{B. Effect on flowering:}

Data of the effect of different hydroponic solutions and media on some flowering traits of Lilium longiflorum, L. cv. Honesty after 50 days are presented in Tables $(18,19,20,21,22,23,24,25$ and 26) as showed that Hoagland solution with Hundz-soil as a hydroponic medium recorded the highest values in most treatments.

Many authors referred to the importance of hydroponic solutions on accelerating flowering (Shi et al.,( 1993); Viti and Cinelli, (1993); Coetzer et al., (1994); Teragishi et al., (1998); Sotiropoulos et al., (1998);; Teragishi et al.,(2000); Sotiropoulos et al., (2003); Doncheva et al., (2006); Saqib et al., (2006) and Ortega-Blu et al. (2006) as reported that fertigation is a necessary management practice in hydroponic Lilium production systems because nutrients supplied by the mother bulb are not sufficient to complete the production cycle; Şirin (2011) declared that nutrition has a great importance for plant growth and yield in soilless culture whereas all the essential elements are supplied to the plants by dissolving fertilizer salts in water to make up the nutrient solution;Asker (2015) indicated that number and quality of flowers were influenced by different nutrient solutions even so the flowers of all treatments were in a good quality and Al-Ajlouni et al. (2017) studied Asiatic hybrid Lilium (Lilium $\times$ elegans Thunb.) cv. 'Fangio') under the soilless culture. 
Table 18: Effect of different nutrient solutions and different media on flower bud initiation (days) of Asiatic lily (Lilium longifloum cv. Honesty) in two seasons (2017/2018 and 2018/2019).

\begin{tabular}{|c|c|c|c|c|c|c|c|c|}
\hline \multirow{3}{*}{$\begin{array}{c}\text { Treatments } \\
\text { Media }\end{array}$} & \multicolumn{8}{|c|}{ Flower bud initiation (days) } \\
\hline & \multicolumn{4}{|c|}{ First season $(2017 / 2018)$} & \multicolumn{4}{|c|}{ Second season $(2018 / 2019)$} \\
\hline & Gravels & Vermiculite & $\begin{array}{c}\text { Hundz- } \\
\text { soil }\end{array}$ & Mean & Gravels & Vermiculite & $\begin{array}{c}\text { Hundz- } \\
\text { soil }\end{array}$ & Mean \\
\hline Water & $47.12 \mathrm{a}$ & $44.57 \mathrm{~b}$ & $43.52 \mathrm{c}$ & 45.07 A & $45.17 \mathrm{a}$ & $43.58 \mathrm{~b}$ & $41.65 \mathrm{c}$ & $43.47 \mathrm{~A}$ \\
\hline B.F. & $42.15 \mathrm{~d}$ & $39.25 \mathrm{e}$ & $37.41 \mathrm{n}$ & 39.61 B & $40.65 \mathrm{~d}$ & $37.25 \mathrm{e}$ & $35.85 \mathrm{o}$ & $37.92 \mathrm{~B}$ \\
\hline M.S. & $39.13 \mathrm{f}$ & $38.15 \mathrm{i}$ & $37.79 \mathrm{k}$ & $38.35 \mathrm{C}$ & $37.15 \mathrm{f}$ & 36.171 & $35.90 \mathrm{n}$ & $36.41 \mathrm{C}$ \\
\hline Hoag. & $38.12 \mathrm{j}$ & $37.52 \mathrm{~m}$ & $34.75 \mathrm{r}$ & $36.80 \mathrm{~F}$ & $36.20 \mathrm{k}$ & $36.24 \mathrm{j}$ & $34.00 \mathrm{r}$ & $35.48 \mathrm{~F}$ \\
\hline Hoag. +Si. & $38.35 \mathrm{~h}$ & 37.621 & $35.79 \mathrm{q}$ & 37.25 D & $36.59 \mathrm{~h}$ & $36.99 \mathrm{~g}$ & $34.79 \mathrm{q}$ & 36.12 D \\
\hline Hoag. + BF. & $37.15 \mathrm{p}$ & $37.35 \mathrm{o}$ & $38.75 \mathrm{~g}$ & $37.75 \mathrm{E}$ & $36.12 \mathrm{~m}$ & $36.37 \mathrm{i}$ & $35.11 \mathrm{p}$ & $35.87 \mathrm{E}$ \\
\hline Mean & $40.34 \mathrm{~A}$ & 39.08 B & $38.00 \mathrm{C}$ & & $38.65 \mathrm{~A}$ & 37.77 B & 36.22 C & \\
\hline
\end{tabular}

Means followed by the same letters in a column or raw do not differ significantly according to Duncan's New Multiple Range test at $\mathrm{P}=0.05$.

Table 19: Effect of different nutrient solutions and different media on flowering bud length (cm) of Asiatic lily (Lilium longifloum cv. Honesty) in two seasons (2017/2018 and 2018/2019).

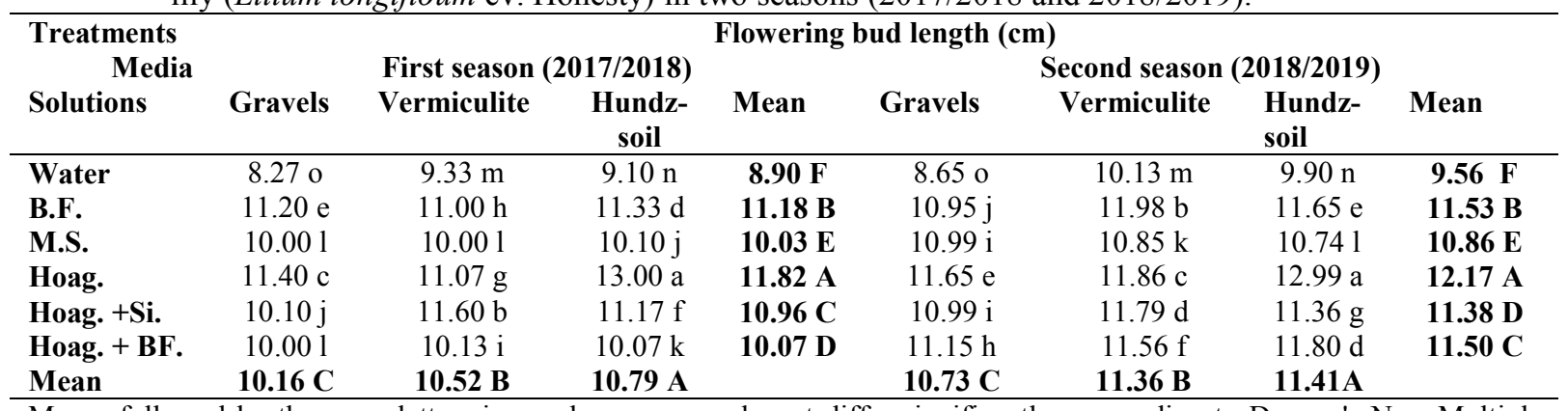

Means followed by the same letters in a column or raw do not differ significantly according to Duncan's New Multiple Range test at $\mathrm{P}=0.05$.

Table 20: Effect of different nutrient solutions and different media on flowering time (days) of Asiatic lily (Lilium longifloum cv. Honesty) in two seasons (2017/2018 and 2018/2019).

\begin{tabular}{|c|c|c|c|c|c|c|c|c|}
\hline \multirow{3}{*}{$\begin{array}{l}\text { Treatments } \\
\text { Media } \\
\text { Solutions } \\
\end{array}$} & \multicolumn{8}{|c|}{ Flowering time (days) } \\
\hline & \multicolumn{4}{|c|}{ First season $(2017 / 2018)$} & \multicolumn{4}{|c|}{ Second season $(2018 / 2019)$} \\
\hline & Gravels & Vermiculite & $\begin{array}{c}\text { Hundz- } \\
\text { soil }\end{array}$ & Mean & Gravels & Vermiculite & Hundz- soil & Mean \\
\hline Water & $61.68 \mathrm{~b}$ & $63.02 \mathrm{a}$ & $60.01 \mathrm{c}$ & $61.57 \mathrm{~A}$ & $62.11 \mathrm{a}$ & $60.76 \mathrm{~b}$ & $59.13 \mathrm{c}$ & $60.67 \mathrm{~A}$ \\
\hline B.F. & $59.67 \mathrm{~d}$ & $58.02 \mathrm{e}$ & 54.011 & $57.23 \mathrm{~B}$ & $56.57 \mathrm{e}$ & $56.12 \mathrm{f}$ & $56.67 \mathrm{~d}$ & 56.45 B \\
\hline M.S. & $56.11 \mathrm{~g}$ & $56.00 \mathrm{~h}$ & $53.33 \mathrm{n}$ & 55.15 D & $55.86 \mathrm{~g}$ & $54.21 \mathrm{j}$ & $54.00 \mathrm{~m}$ & $54.69 \mathrm{C}$ \\
\hline Hoag. & $55.67 \mathrm{i}$ & $53.00 \mathrm{o}$ & $50.03 \mathrm{q}$ & 52.90 F & $55.12 \mathrm{~h}$ & $54.13 \mathrm{k}$ & $52.36 \mathrm{r}$ & 53.87 D \\
\hline Hoag. +Si. & $55.67 \mathrm{i}$ & $53.67 \mathrm{~m}$ & $52.00 \mathrm{p}$ & 53.78 E & 54.111 & $53.06 \mathrm{p}$ & $53.62 \mathrm{n}$ & 53.60 F \\
\hline Hoag. + BF. & $56.84 \mathrm{f}$ & $54.67 \mathrm{k}$ & $55.01 \mathrm{j}$ & $55.51 \mathrm{C}$ & $54.85 \mathrm{i}$ & $53.13 \mathrm{o}$ & $53.00 \mathrm{q}$ & $53.66 \mathrm{E}$ \\
\hline Mean & $57.61 \mathrm{~A}$ & 56.34 B & 54.07 C & & $56.44 \mathrm{~A}$ & 55.24 B & $54.80 \mathrm{C}$ & \\
\hline
\end{tabular}

Means followed by the same letters in a column or raw do not differ significantly according to Duncan's New Multiple Range test at $\mathrm{P}=0.05$.

Table 21: Effect of different solutions and different media on flower stalk length (cm) of Asiatic lily (Lilium longifloum cv. Honesty) in two seasons (2017/2018 and 2018/2019).

\begin{tabular}{|c|c|c|c|c|c|c|c|c|}
\hline \multirow{3}{*}{$\begin{array}{l}\text { Treatments } \\
\text { Media } \\
\text { Solutions }\end{array}$} & \multicolumn{8}{|c|}{$\begin{array}{ll}\text { Flower stalk length }(\mathrm{cm}) \\
\end{array}$} \\
\hline & \multicolumn{4}{|c|}{ First season $(2017 / 2018)$} & \multicolumn{4}{|c|}{ Second season $(2018 / 2019)$} \\
\hline & Gravels & Vermiculite & $\begin{array}{c}\text { Hundz- } \\
\text { soil }\end{array}$ & Mean & Gravels & Vermiculite & $\begin{array}{c}\text { Hundz- } \\
\text { soil }\end{array}$ & Mean \\
\hline Water & $5.97 \mathrm{q}$ & $6.10 \mathrm{p}$ & $6.80 \mathrm{o}$ & $6.29 \mathrm{~A}$ & $6.53 \mathrm{r}$ & $7.65 \mathrm{q}$ & $8.00 \mathrm{p}$ & $7.39 \mathrm{~F}$ \\
\hline B.F. & $8.10 \mathrm{n}$ & 8.701 & $10.90 \mathrm{~b}$ & $9.23 \mathrm{D}$ & $9.50 \mathrm{o}$ & $9.80 \mathrm{n}$ & $10.97 \mathrm{~d}$ & $10.09 \mathrm{D}$ \\
\hline M.S. & $8.37 \mathrm{~m}$ & $9.27 \mathrm{j}$ & $9.00 \mathrm{k}$ & 8.88 E & 9.881 & $9.85 \mathrm{~m}$ & $9.90 \mathrm{k}$ & $9.87 \mathrm{E}$ \\
\hline Hoag. & $10.10 \mathrm{c}$ & $10.03 \mathrm{~d}$ & $11.00 \mathrm{a}$ & $10.38 \mathrm{~A}$ & $10.23 \mathrm{~g}$ & $10.10 \mathrm{j}$ & $11.55 \mathrm{a}$ & $10.92 A$ \\
\hline Hoag. +Si. & $9.00 \mathrm{k}$ & $10.00 \mathrm{e}$ & $9.33 \mathrm{i}$ & $9.44 \mathrm{C}$ & $10.16 \mathrm{~h}$ & $10.12 \mathrm{i}$ & $10.34 \mathrm{f}$ & $10.21 \mathrm{C}$ \\
\hline Hoag. + BF. & $9.53 \mathrm{~h}$ & $9.93 \mathrm{f}$ & $9.67 \mathrm{~g}$ & 9.71 B & $10.53 \mathrm{e}$ & $10.99 \mathrm{c}$ & $11.02 \mathrm{~b}$ & $10.85 \mathrm{~B}$ \\
\hline Mean & $8.51 \mathrm{C}$ & $9.01 \mathrm{~B}$ & $9.45 \mathrm{~A}$ & & $9.47 \mathrm{C}$ & $9.90 \mathrm{~B}$ & $10.30 \mathrm{~A}$ & \\
\hline
\end{tabular}

Means followed by the same letters in a column or raw do not differ significantly according to Duncan's New Multiple Range test at $\mathrm{P}=0.05$. 
Table 22: Effect of different solutions and different media on flower stem diameter (cm) of Asiatic lily (Lilium longifloum cv. Honesty) in two seasons (2017/2018 and 2018/2019).

\begin{tabular}{|c|c|c|c|c|c|c|c|c|}
\hline \multirow{3}{*}{$\begin{array}{l}\text { Treatments } \\
\text { Media }\end{array}$} & \multicolumn{8}{|c|}{\begin{tabular}{|l} 
Flower stem diameter $(\mathrm{cm})$ \\
\end{tabular}} \\
\hline & \multicolumn{4}{|c|}{ First season $(2017 / 2018)$} & \multicolumn{4}{|c|}{ Second season $(2018 / 2019)$} \\
\hline & Gravels & Vermiculite & $\begin{array}{l}\text { Hundz- } \\
\text { soil }\end{array}$ & Mean & Gravels & Vermiculite & $\begin{array}{l}\text { Hundz- } \\
\text { soil }\end{array}$ & Mean \\
\hline Water & $0.51 \mathrm{f}$ & $0.52 \mathrm{f}$ & $0.58 \mathrm{e}$ & $0.55 \mathrm{~F}$ & $0.50 \mathrm{~h}$ & $0.54 \mathrm{~g}$ & $0.59 \mathrm{f}$ & $0.54 \mathrm{D}$ \\
\hline B.F. & $0.70 \mathrm{c}$ & $0.70 \mathrm{c}$ & $0.70 \mathrm{c}$ & $0.70 \mathrm{D}$ & $0.71 \mathrm{de}$ & $0.73 \mathrm{~cd}$ & $0.74 \mathrm{bc}$ & $0.73 \mathrm{~B}$ \\
\hline M.S. & $0.66 \mathrm{~d}$ & $0.66 \mathrm{~d}$ & $0.66 \mathrm{~d}$ & $0.66 \mathrm{C}$ & $0.70 \mathrm{e}$ & $0.72 \mathrm{~cd}$ & $0.73 \mathrm{~cd}$ & $0.72 \mathrm{C}$ \\
\hline Hoag. & $0.75 \mathrm{~b}$ & $0.75 \mathrm{~b}$ & $0.79 \mathrm{a}$ & $0.76 \mathrm{~A}$ & $0.73 \mathrm{~cd}$ & $0.74 \mathrm{bc}$ & $0.76 \mathrm{a}$ & $0.74 \mathrm{~A}$ \\
\hline Hoag. +Si. & $0.71 \mathrm{c}$ & $0.75 \mathrm{~b}$ & $0.71 \mathrm{c}$ & 0.72 B & $0.73 \mathrm{~cd}$ & $0.74 \mathrm{bc}$ & $0.76 \mathrm{a}$ & $0.74 \mathrm{~A}$ \\
\hline Hoag. + BF. & $0.71 \mathrm{c}$ & $0.71 \mathrm{c}$ & $0.71 \mathrm{c}$ & $0.71 \mathrm{~B}$ & $0.73 \mathrm{~cd}$ & $0.73 \mathrm{~cd}$ & $0.75 \mathrm{ab}$ & $0.74 \mathrm{~A}$ \\
\hline Mean & $0.67 \mathrm{C}$ & 0.68 B & $0.70 \mathrm{~A}$ & & $0.68 \mathrm{C}$ & $0.70 \mathrm{~B}$ & $0.72 \mathrm{~A}$ & \\
\hline
\end{tabular}

Means followed by the same letters in a column or raw do not differ significantly according to Duncan's New Multiple Range test at $\mathrm{P}=0.05$.

Table 23: Effect of different nutrient solutions and different media on inflorescence diameter (cm) of Asiatic lily (Lilium longifloum cv. Honesty) in the two seasons (2017/2018 and 2018/2019).

\begin{tabular}{|c|c|c|c|c|c|c|c|c|}
\hline \multirow{3}{*}{$\begin{array}{l}\text { Treatments } \\
\text { Media } \\
\text { Solutions }\end{array}$} & \multicolumn{8}{|c|}{ Inflorescence diameter ( cm) } \\
\hline & \multicolumn{4}{|c|}{ First season $(2017 / 2018)$} & \multicolumn{4}{|c|}{ Second season $(2018 / 2019)$} \\
\hline & Gravels & Vermiculite & $\begin{array}{c}\text { Hundz- } \\
\text { soil }\end{array}$ & Mean & Gravels & Vermiculite & $\begin{array}{c}\text { Hundz- } \\
\text { soil }\end{array}$ & Mean \\
\hline Water & 14.10 o & $15.65 \mathrm{n}$ & $16.30 \mathrm{k}$ & $15.35 \mathrm{~F}$ & $16.90 \mathrm{p}$ & $17.65 \mathrm{o}$ & 18.651 & $17.74 \mathrm{~F}$ \\
\hline B.F. & $16.10 \mathrm{~m}$ & 16.131 & $19.00 \mathrm{c}$ & 17.08 D & $17.87 \mathrm{n}$ & 18.651 & $20.65 \mathrm{e}$ & $19.06 \mathrm{E}$ \\
\hline M.S. & $16.10 \mathrm{~m}$ & $17.27 \mathrm{~h}$ & $17.03 \mathrm{i}$ & $16.80 \mathrm{E}$ & $17.99 \mathrm{~m}$ & 18.651 & $20.76 \mathrm{~d}$ & 19.13 D \\
\hline Hoag. & $17.97 \mathrm{~g}$ & $18.00 \mathrm{f}$ & $19.97 \mathrm{a}$ & $18.65 \mathrm{~A}$ & $18.96 \mathrm{k}$ & $19.65 \mathrm{~h}$ & $21.92 \mathrm{a}$ & $20.17 \mathrm{~A}$ \\
\hline Hoag. +Si. & $17.00 \mathrm{j}$ & $18.97 \mathrm{~d}$ & $19.00 \mathrm{c}$ & $18.32 \mathrm{~B}$ & $19.87 \mathrm{f}$ & $19.10 \mathrm{j}$ & $21.15 \mathrm{c}$ & $20.04 \mathrm{C}$ \\
\hline Hoag. + BF. & $17.03 \mathrm{i}$ & $18.03 \mathrm{e}$ & $19.45 \mathrm{~b}$ & $18.17 \mathrm{C}$ & $19.75 \mathrm{~g}$ & $19.12 \mathrm{i}$ & $21.65 \mathrm{~b}$ & $20.17 \mathrm{AB}$ \\
\hline Mean & $16.38 \mathrm{C}$ & $17.34 \mathrm{~B}$ & $18.46 \mathrm{~A}$ & & $18.55 \mathrm{C}$ & $18.81 \mathrm{~b}$ & $20.80 \mathrm{~A}$ & \\
\hline
\end{tabular}

Means followed by the same letters in a column or raw do not differ significantly according to Duncan's New Multiple Range test at $\mathrm{P}=0.05$.

Table 24: Effect of different nutrient solutions and different media on inflorescences number of Asiatic lily (Lilium longifloum cv. Honesty) in two seasons (2017/2018 and 2018/2019).

\begin{tabular}{|c|c|c|c|c|c|c|c|c|}
\hline \multirow{3}{*}{$\begin{array}{l}\text { Treatments } \\
\text { Media } \\
\text { Solutions }\end{array}$} & \multicolumn{8}{|c|}{ Number of inflorescences /plant } \\
\hline & \multicolumn{4}{|c|}{ First season $(2017 / 2018)$} & \multicolumn{4}{|c|}{ Second season $(2018 / 2019)$} \\
\hline & Gravels & Vermiculite & $\begin{array}{c}\text { Hundz- } \\
\text { soil }\end{array}$ & Mean & Gravels & Vermiculite & $\begin{array}{c}\text { Hundz- } \\
\text { soil }\end{array}$ & Mean \\
\hline Water & $5.10 \mathrm{k}$ & $6.00 \mathrm{i}$ & $5.50 \mathrm{j}$ & $5.53 \mathrm{C}$ & $5.00 \mathrm{~m}$ & $5.98 \mathrm{k}$ & 5.581 & $5.52 \mathrm{~F}$ \\
\hline B.F. & $6.40 \mathrm{c}$ & 6.00 & $6.40 \mathrm{c}$ & $6.27 \mathrm{~A}$ & $6.13 \mathrm{j}$ & $6.32 \mathrm{~h}$ & $6.98 \mathrm{a}$ & $6.48 \mathrm{E}$ \\
\hline M.S. & $6.10 \mathrm{~g}$ & $6.43 \mathrm{~b}$ & $6.07 \mathrm{~h}$ & $6.20 \mathrm{~B}$ & $6.22 \mathrm{i}$ & $6.46 \mathrm{f}$ & $6.79 \mathrm{c}$ & $6.49 \mathrm{D}$ \\
\hline Hoag. & $6.13 \mathrm{f}$ & $6.17 \mathrm{e}$ & $6.47 \mathrm{a}$ & $6.26 \mathrm{~A}$ & $6.45 \mathrm{f}$ & $6.51 \mathrm{e}$ & $6.99 \mathrm{a}$ & $6.65 \mathrm{~A}$ \\
\hline Hoag. +Si. & $6.10 \mathrm{~g}$ & $6.00 \mathrm{i}$ & $6.47 \mathrm{a}$ & $6.19 \mathrm{~B}$ & $6.34 \mathrm{~g}$ & $6.51 \mathrm{e}$ & $6.85 \mathrm{~b}$ & $6.57 \mathrm{~B}$ \\
\hline Hoag. + BF. & $6.30 \mathrm{~d}$ & $6.10 \mathrm{~g}$ & $6.17 \mathrm{e}$ & $6.19 \mathrm{~B}$ & $6.31 \mathrm{~h}$ & $6.51 \mathrm{e}$ & $6.68 \mathrm{~d}$ & $6.50 \mathrm{C}$ \\
\hline Mean & $6.02 \mathrm{C}$ & $6.12 \mathrm{~B}$ & $6.18 \mathrm{~A}$ & & $6.08 \mathrm{C}$ & $6.38 \mathrm{~B}$ & $6.65 \mathrm{~A}$ & \\
\hline
\end{tabular}

Table 25: Effect of different nutrient solutions and different media on vase life / plant (days) of Asiatic lily (Lilium longifloum cv. Honesty) in two seasons (2017/2018 and 2018/2019).

\begin{tabular}{|c|c|c|c|c|c|c|c|c|}
\hline \multirow{3}{*}{$\begin{array}{l}\text { Treatments } \\
\text { Media } \\
\text { Solutions }\end{array}$} & \multicolumn{8}{|c|}{ Vase life / plant ( days) } \\
\hline & \multicolumn{4}{|c|}{ First season $(2017 / 2018)$} & \multicolumn{4}{|c|}{ Second season $(2018 / 2019)$} \\
\hline & Gravels & Vermiculite & $\begin{array}{c}\text { Hundz- } \\
\text { soil }\end{array}$ & Mean & Gravels & Vermiculite & $\begin{array}{c}\text { Hundz- } \\
\text { soil }\end{array}$ & Mean \\
\hline Water & $10.56 \mathrm{r}$ & $12.26 \mathrm{q}$ & $13.70 \mathrm{o}$ & $12.17 \mathrm{E}$ & $11.46 \mathrm{q}$ & $12.89 \mathrm{p}$ & $13.90 \mathrm{n}$ & $12.75 \mathrm{~A}$ \\
\hline B.F. & $13.25 \mathrm{p}$ & $14.90 \mathrm{~h}$ & $14.65 \mathrm{j}$ & 14.30 D & $13.99 \mathrm{~m}$ & $14.27 \mathrm{i}$ & $14.12 \mathrm{k}$ & $14.14 \mathrm{E}$ \\
\hline M.S. & $14.86 \mathrm{i}$ & $15.18 \mathrm{~d}$ & $15.47 \mathrm{c}$ & $15.17 \mathrm{~A}$ & 14.101 & $15.12 \mathrm{f}$ & $15.30 \mathrm{~d}$ & 15.14 B \\
\hline Hoag. & 14.361 & $15.12 \mathrm{f}$ & $15.99 \mathrm{a}$ & $15.16 \mathrm{~A}$ & $14.14 \mathrm{j}$ & $15.68 \mathrm{~b}$ & $15.92 \mathrm{a}$ & $15.25 \mathrm{~A}$ \\
\hline Hoag. +Si. & $14.05 \mathrm{n}$ & $15.16 \mathrm{e}$ & $15.75 \mathrm{~b}$ & $14.99 \mathrm{~B}$ & $13.10 \mathrm{o}$ & $15.03 \mathrm{~h}$ & $15.23 \mathrm{e}$ & 14.75 D \\
\hline Hoag. + BF. & $14.62 \mathrm{k}$ & $14.10 \mathrm{~m}$ & $15.10 \mathrm{~g}$ & $14.91 \mathrm{C}$ & $14.00 \mathrm{~m}$ & $15.09 \mathrm{~g}$ & $15.41 \mathrm{c}$ & $14.83 \mathrm{C}$ \\
\hline Mean & $13.62 \mathrm{C}$ & $14.62 \mathrm{~B}$ & 15.11 AB & & $13.76 \mathrm{C}$ & 14.68B & $15.98 \mathrm{~A}$ & \\
\hline
\end{tabular}

Means followed by the same letters in a column or raw do not differ significantly according to Duncan's New Multiple Range test at $\mathrm{P}=0.05$. 
Table 26: Effect of the best media, solution and their interaction on some flowering traits of Asiatic lily (Lilium longifloum cv. Honesty) in two seasons (2017/2018 and 2018/2019).

\begin{tabular}{|c|c|c|c|c|c|c|c|c|}
\hline \multirow[t]{2}{*}{ Treatments } & \multicolumn{2}{|c|}{$\begin{array}{c}\text { Flower bud } \\
\text { initiation } \\
\text { (days) }\end{array}$} & \multicolumn{2}{|c|}{$\begin{array}{c}\text { Flowering bud } \\
\text { length } \\
(\mathrm{cm})\end{array}$} & \multicolumn{2}{|c|}{$\begin{array}{l}\text { Flowering time } \\
\text { (days) }\end{array}$} & \multicolumn{2}{|c|}{$\begin{array}{c}\text { Flower stalk } \\
\text { length } \\
(\mathrm{cm})\end{array}$} \\
\hline & 1st. & 2nd. & 1st. & 2nd. & 1st. & 2nd. & 1st. & 2nd. \\
\hline Water (control) & 45.07 & 43.47 & 8.90 & 9.56 & 61.57 & 60.67 & 6.29 & 7.39 \\
\hline Hoag. solution & 36.80 & 35.48 & 11.82 & 12.17 & 52.90 & 53.87 & 10.38 & 10.92 \\
\hline Gravels & 40.34 & 38.65 & 10.16 & 10.73 & 57.61 & 56.44 & 8.51 & 9.47 \\
\hline Hundz-soil & 38.00 & 36.23 & 10.79 & 11.41 & 54.07 & 54.80 & 9.45 & 10.30 \\
\hline Interaction (Hoag.+Hundz soil). & 34.75 & 34.00 & 13.00 & 12.99 & 50.03 & 52.36 & 11.00 & 11.55 \\
\hline
\end{tabular}

$1^{\text {st }}=$ First season and 2 nd. $=$ Second season.

Table 26: Continued

\begin{tabular}{|c|c|c|c|c|c|c|c|c|}
\hline \multirow[t]{2}{*}{ Treatments } & \multicolumn{2}{|c|}{$\begin{array}{l}\text { Flower stem } \\
\text { diameter } \\
(\mathrm{cm})\end{array}$} & \multicolumn{2}{|c|}{$\begin{array}{l}\text { Inflorescence } \\
\text { diameter } \\
(\mathrm{cm})\end{array}$} & \multicolumn{2}{|c|}{$\begin{array}{c}\text { Number of } \\
\text { inflorescence/ } \\
\text { plant }\end{array}$} & \multicolumn{2}{|c|}{$\begin{array}{l}\text { Vase life } \\
\text { / plant } \\
\text { ( days) }\end{array}$} \\
\hline & $1^{\text {st. }}$ & $2^{\text {nd. }}$ & $1^{\text {st. }}$ & $2^{\text {nd. }}$ & $1^{\text {st. }}$ & $2^{\text {nd. }}$ & $1^{\text {st. }}$ & $2^{\text {nd. }}$ \\
\hline Water (control) & 0.55 & 0.54 & 15.35 & 17.75 & 5.53 & 5.52 & 12.17 & 12.75 \\
\hline Hoag. solution & 0.76 & 0.74 & 18.65 & 20.17 & 6.26 & 6.66 & 15.16 & 15.25 \\
\hline Gravels & 0.67 & 0.68 & 16.38 & 18.55 & 6.02 & 6.08 & 13.62 & 13.76 \\
\hline Hundz-soil & 0.70 & 0.72 & 18.46 & 20.80 & 6.18 & 6.65 & 15.11 & 15.98 \\
\hline Interaction (Hoag.+Hundz soil) & 0.79 & 0.76 & 19.97 & 21.92 & 6.47 & 6.99 & 15.99 & 15.92 \\
\hline
\end{tabular}

$\mathbf{1}^{\text {st }}$ = First season and 2nd.= Second season.

\section{Effect on chemical composition:}

Data of the effect growth treatments on some chemical traits of Lilium longiflorum L.cv. Honesty are presented after 50 days in Tables $(27,28,29,30$ and 31) showed that Hoagland solution with Hundz-soil recorded the highest values in most treatments in two seasons. The increase in all macro elements in Lilium plants may be due to the stimulatory influence of applied Hoag. Solution and Hundz-soil on the vegetative growth and flowering as mentioned before.

Table 27: Effect of different nutrient solutions and different media on total chlorophyll in the leaves $(\mathrm{mg} / \mathrm{g}$ f.w.) of Asiatic lily (Lilium longifloum cv. Honesty) in two seasons (2017/2018 and 2018/2019).

\begin{tabular}{|c|c|c|c|c|c|c|c|c|}
\hline \multirow{3}{*}{$\begin{array}{r}\text { Treatments } \\
\text { Media }\end{array}$} & \multicolumn{8}{|c|}{ Total chlorophyll in leaves (mg/g f.w.) } \\
\hline & \multicolumn{4}{|c|}{ First season $(2017 / 2018)$} & \multicolumn{4}{|c|}{ Second season $(2018 / 2019)$} \\
\hline & Gravels & Vermiculite & $\begin{array}{l}\text { Hundz- } \\
\text { soil }\end{array}$ & Mean & Gravels & Vermiculite & $\begin{array}{l}\text { Hundz- } \\
\text { soil }\end{array}$ & Mean \\
\hline Water & $1.05 \mathrm{~m}$ & 1.181 & $1.39 \mathrm{k}$ & $1.21 \mathrm{~A}$ & $1.03 \mathrm{p}$ & $1.17 \mathrm{o}$ & 1.391 & $1.20 \mathrm{~F}$ \\
\hline B.F. & $1.84 \mathrm{~h}$ & $1.98 \mathrm{f}$ & $2.07 \mathrm{e}$ & $1.97 \mathrm{D}$ & $1.69 \mathrm{i}$ & $1.86 \mathrm{f}$ & $2.07 \mathrm{c}$ & $1.88 \mathrm{C}$ \\
\hline M.S. & $1.75 \mathrm{j}$ & $1.92 \mathrm{~g}$ & $2.23 \mathrm{~d}$ & $1.97 \mathrm{D}$ & $1.63 \mathrm{j}$ & $1.82 \mathrm{~g}$ & $2.23 \mathrm{~b}$ & $1.89 \mathrm{~B}$ \\
\hline Hoag. & $2.06 \mathrm{e}$ & $2.23 \mathrm{~d}$ & $2.50 \mathrm{a}$ & $2.26 \mathrm{~A}$ & $1.76 \mathrm{~h}$ & $1.88 \mathrm{e}$ & $2.29 \mathrm{a}$ & $1.98 \mathrm{~A}$ \\
\hline Hoag. +Si. & $1.80 \mathrm{i}$ & $2.22 \mathrm{~d}$ & $2.44 \mathrm{~b}$ & $2.16 \mathrm{~B}$ & $1.32 \mathrm{~m}$ & $1.98 \mathrm{~d}$ & $2.29 \mathrm{a}$ & $1.86 \mathrm{D}$ \\
\hline Hoag. + BF. & $1.80 \mathrm{i}$ & $1.98 \mathrm{f}$ & $2.28 \mathrm{c}$ & $2.02 \mathrm{C}$ & $1.30 \mathrm{n}$ & $1.45 \mathrm{k}$ & $2.22 \mathrm{~b}$ & $1.66 \mathrm{E}$ \\
\hline Mean & $1.17 \mathrm{C}$ & $1.92 \mathrm{~B}$ & $2.15 \mathrm{~A}$ & & $1.47 \mathrm{C}$ & $1.69 \mathrm{~B}$ & $2.08 \mathrm{~A}$ & \\
\hline
\end{tabular}

Means followed by the same letter/s in a column or raw do not differ significantly according to Duncan's New Multiple Range test at $\mathrm{P}=0.05$.

Table 28: Effect of different nutrient solutions and different medium on N\% in the leaves Of Asiatic lily (Lilium longifloum cv. Honesty) in two seasons (2017/2018 and 2018/2019).

\begin{tabular}{|c|c|c|c|c|c|c|c|c|}
\hline \multirow{3}{*}{$\begin{array}{l}\begin{array}{l}\text { Treatments } \\
\text { Media }\end{array} \\
\text { Solutions }\end{array}$} & \multicolumn{8}{|c|}{$\mathrm{N} \%$ in the leaves } \\
\hline & \multicolumn{4}{|c|}{ First season $(2017 / 2018)$} & \multicolumn{4}{|c|}{ Second season $(2018 / 2019)$} \\
\hline & Gravels & Vermiculite & $\begin{array}{c}\text { Hundz- } \\
\text { soil }\end{array}$ & Mean & Gravels & Vermiculite & $\begin{array}{c}\text { Hundz- } \\
\text { soil }\end{array}$ & Mean \\
\hline Water & $1.27 \mathrm{r}$ & $1.76 \mathrm{q}$ & $2.24 \mathrm{p}$ & $1.76 \mathrm{~F}$ & $1.28 \mathrm{n}$ & $1.76 \mathrm{~m}$ & 2.251 & $1.76 \mathrm{~F}$ \\
\hline B.F. & $2.64 n$ & $3.34 \mathrm{k}$ & $3.44 \mathrm{o}$ & $3.14 \mathrm{E}$ & 2.251 & $3.35 \mathrm{j}$ & $3.47 \mathrm{~h}$ & $3.02 \mathrm{E}$ \\
\hline M.S. & $3.41 \mathrm{j}$ & $3.63 \mathrm{f}$ & $3.74 \mathrm{e}$ & $3.95 \mathrm{~A}$ & $3.48 \mathrm{~h}$ & $3.69 \mathrm{e}$ & $3.79 \mathrm{c}$ & $3.65 \mathrm{~B}$ \\
\hline Hoag. & 3.311 & $4.02 \mathrm{~b}$ & $4.44 \mathrm{a}$ & $3.92 \mathrm{~B}$ & $3.35 \mathrm{j}$ & $4.02 \mathrm{~b}$ & $4.42 \mathrm{a}$ & $3.93 \mathrm{~A}$ \\
\hline Hoag. +Si. & $2.98 \mathrm{~m}$ & $3.51 \mathrm{~h}$ & $3.61 \mathrm{~g}$ & $3.37 \mathrm{D}$ & $2.91 \mathrm{k}$ & $3.52 \mathrm{~g}$ & $3.62 \mathrm{f}$ & $3.35 \mathrm{D}$ \\
\hline Hoag. + BF. & $3.44 \mathrm{i}$ & $3.86 \mathrm{c}$ & $3.78 \mathrm{~d}$ & $3.69 \mathrm{C}$ & $3.42 \mathrm{i}$ & $3.75 \mathrm{~d}$ & $3.78 \mathrm{c}$ & $3.65 \mathrm{C}$ \\
\hline Mean & $2.84 \mathrm{C}$ & $3.35 \mathrm{~B}$ & $3.54 \mathrm{~A}$ & & $2.78 \mathrm{C}$ & $3.35 \mathrm{~B}$ & $3.55 \mathrm{~A}$ & \\
\hline
\end{tabular}

Means followed by the same letters in a column or raw do not differ significantly according to Duncan's New Multiple Range test at $\mathrm{P}=0.05$. 
Table 29: Effect of different solutions and different media on $\mathrm{P} \%$ in the leaves of Asiatic lily (Lilium longifloum cv. Honesty) in two seasons (2017/2018 and 2018/2019).

Treatments
Media

First season (2017/2018) $\mathrm{P} \%$ in the leaves

Solutions

\begin{tabular}{|c|c|c|c|c|c|c|c|c|}
\hline Solutions & Gravels & Vermiculite & $\begin{array}{c}\text { Hundz- } \\
\text { soil }\end{array}$ & Mean & Gravels & Vermiculite & $\begin{array}{l}\text { Hundz- } \\
\text { soil }\end{array}$ & Mean \\
\hline Water & $0.11 \mathrm{k}$ & $0.13 \mathrm{ij}$ & $0.14 \mathrm{hi}$ & $0.13 \mathrm{E}$ & $0.12 \mathrm{j}$ & $0.13 \mathrm{ij}$ & 0.14 hi & $0.13 \mathrm{D}$ \\
\hline B.F. & $0.12 \mathrm{jk}$ & $0.16 \mathrm{fg}$ & $0.23 \mathrm{bc}$ & $0.17 \mathrm{C}$ & $0.12 \mathrm{j}$ & $0.16 \mathrm{i}$ & $0.23 \mathrm{~b}$ & $0.17 \mathrm{C}$ \\
\hline M.S. & $0.12 \mathrm{jk}$ & $0.15 \mathrm{gh}$ & $0.22 \mathrm{c}$ & $0.16 \mathrm{D}$ & $0.12 \mathrm{j}$ & $0.15 \mathrm{gh}$ & $0.22 \mathrm{~b}$ & $0.17 \mathrm{C}$ \\
\hline Hoag. & $0.16 \mathrm{fg}$ & $0.19 \mathrm{~d}$ & $0.25 \mathrm{a}$ & $0.20 \mathrm{~A}$ & $0.13 \mathrm{ij}$ & $0.20 \mathrm{c}$ & $0.26 \mathrm{a}$ & 0.19 B \\
\hline Hoag. +Si. & $0.15 \mathrm{gh}$ & $0.18 \mathrm{de}$ & $0.23 \mathrm{bc}$ & 0.19 B & $0.16 \mathrm{fg}$ & $0.18 \mathrm{de}$ & $0.23 \mathrm{~b}$ & 0.19 B \\
\hline Hoag. + BF. & $0.17 \mathrm{ef}$ & $0.18 \mathrm{de}$ & $0.24 \mathrm{ab}$ & $0.20 \mathrm{~A}$ & $0.17 \mathrm{ef}$ & $0.19 \mathrm{~cd}$ & $0.25 \mathrm{a}$ & $0.20 \mathrm{~A}$ \\
\hline Mean & $0.14 \mathrm{C}$ & 0.17 B & $0.22 \mathrm{~A}$ & & $0.14 \mathrm{C}$ & 0.17 B & $0.22 \mathrm{~A}$ & \\
\hline
\end{tabular}

Means followed by the same letters in a column or raw do not differ significantly according to Duncan's New Multiple Range test at $\mathrm{P}=0.05$.

Table 30: Effect of different nutrient solutions and different media on $\mathrm{K} \%$ in the leaves of Asiatic lily (Lilium longifloum cv. Honesty) in two seasons (2017/2018 and 2018/2019).

\begin{tabular}{|c|c|c|c|c|c|c|c|c|}
\hline \multirow{2}{*}{\multicolumn{2}{|c|}{$\begin{array}{r}\text { Treatments } \\
\text { Media }\end{array}$}} & \multicolumn{6}{|c|}{$K \%$ in the leaves } & \\
\hline & & \multicolumn{3}{|c|}{ First season $(2017 / 2018)$} & \multicolumn{4}{|c|}{ Second season $(2018 / 2019)$} \\
\hline Solutions & Gravels & Vermiculite & $\begin{array}{c}\text { Hundz- } \\
\text { soil }\end{array}$ & Mean & Gravels & Vermiculite & $\begin{array}{l}\text { Hundz- } \\
\text { soil }\end{array}$ & Mean \\
\hline Water & $2.34 \mathrm{p}$ & $3.21 \mathrm{k}$ & $3.90 \mathrm{e}$ & $3.15 \mathrm{~F}$ & $2.22 \mathrm{o}$ & $3.01 \mathrm{i}$ & 3.101 & $2.78 \mathrm{~F}$ \\
\hline B.F. & $2.64 n$ & $3.76 \mathrm{f}$ & $4.45 \mathrm{a}$ & $3.62 \mathrm{~B}$ & $2.65 \mathrm{~m}$ & $3.57 \mathrm{~h}$ & $4.32 \mathrm{~d}$ & $3.52 \mathrm{D}$ \\
\hline M.S. & $2.48 \mathrm{o}$ & $3.53 \mathrm{~h}$ & $4.13 \mathrm{~d}$ & $3.38 \mathrm{E}$ & $2.48 \mathrm{n}$ & $3.67 \mathrm{e}$ & $4.32 \mathrm{~d}$ & $3.49 \mathrm{E}$ \\
\hline Hoag. & $2.66 \mathrm{~m}$ & $3.33 \mathrm{i}$ & $4.45 \mathrm{a}$ & $3.48 \mathrm{D}$ & $2.88 \mathrm{k}$ & $3.62 \mathrm{~g}$ & $4.43 \mathrm{~b}$ & $3.64 \mathrm{~A}$ \\
\hline Hoag. +Si. & 2.851 & $3.26 \mathrm{j}$ & $4.41 \mathrm{~b}$ & $3.50 \mathrm{C}$ & $2.66 \mathrm{~m}$ & $3.65 \mathrm{f}$ & $4.46 \mathrm{a}$ & $3.59 \mathrm{C}$ \\
\hline Hoag. + BF. & 2.851 & $3.66 \mathrm{~g}$ & $4.37 \mathrm{c}$ & $3.63 \mathrm{~A}$ & 2.851 & $3.61 \mathrm{~g}$ & $4.36 \mathrm{c}$ & $3.61 \mathrm{~B}$ \\
\hline Mean & $2.63 \mathrm{C}$ & $3.46 \mathrm{~B}$ & $4.28 \mathrm{~A}$ & & $2.62 \mathrm{C}$ & $3.52 \mathrm{~B}$ & 4.17 A & \\
\hline
\end{tabular}

Means followed by the same letters in a column or raw do not differ significantly according to Duncan's New Multiple Range test at $\mathrm{P}=0.05$.

Table 31: Effect of the best media, solution and their interaction on some chemical composition traits of Asiatic lily (Lilium longifloum cv. Honesty) in two seasons (2017/2018 and 2018/2019).

\begin{tabular}{|c|c|c|c|c|c|c|c|c|}
\hline \multirow[t]{2}{*}{ Treatments } & \multicolumn{2}{|c|}{$\begin{array}{c}\text { Total chlorophyll } \\
\text { (mg/g f.w.) }\end{array}$} & \multicolumn{2}{|c|}{$\begin{array}{c}\mathbf{N} \% \\
\text { in leaves }\end{array}$} & \multicolumn{2}{|c|}{$\begin{array}{c}\mathbf{P} \% \\
\text { in leaves }\end{array}$} & \multicolumn{2}{|c|}{$\begin{array}{c}\mathrm{K} \% \\
\text { in leaves }\end{array}$} \\
\hline & 1 st. & 2nd. & 1st. & 2nd. & 1st. & 2nd & 1st. & 2nd. \\
\hline Water (control) & 1.21 & 1.20 & 1.76 & 1.76 & 0.13 & 0.13 & 3.10 & 2.78 \\
\hline Hoag. solution & 2.26 & 1.98 & 3.92 & 3.93 & 0.20 & 0.19 & 3.48 & 3.64 \\
\hline Gravels & 1.17 & 1.47 & 2.84 & 2.78 & 0.14 & 0.14 & 2.63 & 2.62 \\
\hline Hundz-soil & 2.15 & 2.08 & 3.54 & 3.55 & 0.22 & 0.22 & 4.28 & 4.17 \\
\hline Interaction (Hoag.+Hundz -soil) & 2.50 & 2.29 & 4.44 & 4.42 & 0.25 & 0.26 & 4.45 & 4.43 \\
\hline
\end{tabular}

1 St. $=$ First season and $2^{\text {nd. }}=$ Second season.

\section{Acknowledgment}

I extend my deepest gratitude from my heart to Prof. Dr. Magdy A. Eskarous Prof. of Soil Fertility and Plant Nutrition Dept., Water and Environment Res. Inst., ARC Giza, Egypt ; Dr. Emam M.S. Nofal Prof. of Floriculture and Ornamental Hort., Horticulture Department, Fac. Agric. Kafr El-Sheikh, Kafr El-Sheikh Univ. and Prof. Dr. Faisal M. Saadawy Prof. of Floriculture and Ornamental Hort., Horticulture Dept. Horticulture Research Institute, Giza, for their giving the honor of working, continues encouragement and reviewing the manuscript.

\section{Conclusion}

This research proved that Hoagland solution with Hundz-soil under drip hydroponic system gave the best vegetative growth with wonderful flowering characters and the highest daughter bulblets production from cut flowering stem during vase period. 


\section{References}

Al-Ajlouni, M.G., J. Y. Ayad and Y. A. Othman, 2017. Increasing nutrient levels promote growth and flower quality in lilies grown under soilless culture. Hort. Sci. (Prague), 44 (4): 171-177.

Asker, H.M., 2015. Hydroponic technology for lily flowers and bulbs production using rainwater and some common nutrient solutions. Afri. J. Bio., 14(29):2307-2313.

Barnes, j., B.E. Whipker and I. McCall, 2011. Characterization of nutrient disorders of Lilium longiflorum 'Nellie White' and Lilium hybrid 'Brunello'. Proc. II ${ }^{\text {nd }}$ IS on the Genus Lilium Eds.: A. Grassotti and G. Burchi Acta Hort. 900, ISHS 2011.

Bever, A.D., P.A. Ndakidemi and C.P. Laubscher, 2012. Effect of different combinations of Hoagland's solution and Azolla filiculoides on growth and development of Beta vulgaris subsp. cycla 'Fordhook Giant' grown in hydroponic culture. Int. J. Phys. Sci. 7(33): 5281-5287.

Brown, J.D. and O. Lilliland, 1946. Rapid determination of potassium and sodium in plant material and soil extracts by flame photometry. Proc. Amer. Soc. Hort. Sci., 48: 341346.

Coetzer L.A., P.J. Robbertse, R.O. Barnard; E. Tomer and M.F. Simith, 1994. The effect of boron on the growth of mango seedlings. Yearbook South African Mango Growers Association 14: 14-17: $1 \mathrm{pl}$.

Doncheva S., Z. Stoyanova, K. Georgieva; D. Nedeva, R. Dikova, G. Zehirov and A. Nikolova, 2006. Exogenous succinate increases resistance of maize plants to copper stress. J. Plant Nutr. Soil Sci., 169: 247-254.

Hend, N. M. Y.and A.M. Awad, 2015. Economy evaluation of using organic soil conditioner "Hundz soil" and effects on increasing use efficiency of agricultural resources in wheat cultivation on sandy soils. Egypt. J. Agric. Res., 93 (2): 605-622.

Jackson, M.L., 1967. Soil Chemical Analysis. Prentice-Hall Inc. Publisher, Madison, USA.

Kaur, H., S. Rakesh and S. Pankaj, 2016. Effect of Hoagland Solution for Growing Tomato Hydroponically in Greenhouse Hort. Flora Res. Spectrum, 5(4):310-315.

Lincoln, T. and E Zeiger, 2010. Plant Physiology. Fifth Edition. Published by Sinauer Associates, Inc., pp. 92.

Maloupa, E. I., P. Mitsios, F. Martinez and S. Bladenopoulou, 1993. Study of substrates used in Gerbera culture in plastic greenhouse, Acta Hort. (ISHS), 323: 139-144.

Michael J. P., 2002. Vermiculite .U.S. Geological Survey Minerals, p.81-87.

Moran, R., 1982. Formula for determination of chlorophyllous pigments extracted with N, N-dimethylformamide. Plant Physiol., 69: 1376-1381.

MSTAT Computer Program, 1985. Software program for design, management and analysis experimental (version 4.0), Michigan State Uni.

Murashige, T. and F. Skoog, 1962. A revised medium for rapid growth and bio-assay with tobacco tissue culture. Physiolgia Plantarum, 15:473-497.

Neil S. M. and J. H. Lieth, 2007. Modeling Macronutrient Absorption of HydroponicallyGrown Cut Flower Roses. Acta Hort. (ISHS), 751:129-136.

Ortega-Blu R., M. Correa-Benguria and E. Olate-Muñoz, 2006. Determination of nutrient accumulation curves in three cultivars of Lilium spp. for cut flower. Agrociencia, 40: 77-88.

Piper, C.S., 1947. Soil and Plant Analysis. The University of Adelaide, Adeaide, p. 252-275.

Ranjit, S. S., M. Devia, J. Singhb, S. Sharmaa, A. Negia, S. Singha, N. Chauhana, D. Sharmaa and J. C.Sharmaa, 2018. Lettuce Growth and Yield in Hoagland Solution with an Organic Concoction. Int. J. of Veg. Sci., 24(6):557-566.

Safia, H. E., S. S. Helmy, A.M. Abou Dahab, N. E. Metwally and W. R. Hamed, 2018. Soilless culture technique for producing Gladiolus (Gladiolus grandiflorus. Middle East J. Appl. Sci., 8(4):1080-1093.

Saqib M., C. Zörb and S. Schubert, 2006. Salt-resistant and salt-sensitive wheat genotypes show similar biochemical reaction at protein level in the first phase of salt stress. J. Plant Nutr. Soil Sci., 169: 542-548. 
Shi Y., D.H. Byrne; D.W. Reed and R.H. Loeppert, 1993. Influence of bicarbonate level on iron chlorosis development and nutrient uptake of the peach rootstock Montclar. J. Plant Nutr. 16(9):1675-1689.

Şirin, U., 2011. Effects of different nutrient solution formulations on yield and cut flower quality of gerbera (Gerbera jamesonii) grown in soilless culture system. Afr. J. Agric. Res. 6(21), 49104919.

Snedecor, G.W. and W.G. Cochran, 1980. Statistical Methods, 7th ed. Iowa State Univ. Press, Ames, Iowa, U.S.A.

Sotiropoulos T.E., I.N. Therios and K.N. Dimassi, 1998. The effect of toxic boron concentrations on shoot proliferation of in vitro kiwifruit shoot tip culture. Adv. Hortic. Sci., 12(4):196-200.

Sotiropoulos T.E., I.N.Therios and K.N.Dimassi, 2003. Boron toxicity in kiwifruit plants (Actinidia deliciosa), treated with nitrate, ammonium, and a mixture of both. J. Plant Nutr. Soil Sci., 166:529-532.

Sun Y.D., W.R. Luo and H.C. Liu, 2014. Effect of different nitrogen forms on the nutritional quality and physiological characteristics of Chinese chive seedlings. Plant Soil Environ. , 60(5):216220.

Teragishi A., Y. Kanbara and H. Ono, 1998. Effect of low temperature storage, diameter of cuttings and nutrient solution concentration on subsequent growth and fruit set of fig trees grown in hydroponics. J. Jpn. Soc. Hortic. Sci., 67(3):386-390.

Teragishi A, Y. Kanbara and H. Ono, 2000. Effects of foliar application of choline chloride on the quality of winter cropped fig cv. Masui Dauphine grown in hydroponics. J. Jpn. Soc. Hort. Sci., 69(4):390-395.

Thakur, T., 2016. Effect of photoperiod, nitrogen and growing media on morphological development of chrysanthemum (Chrysanthemum morifolium Ramat. Ph.D. Punjab Agric Uni.

Troug, E. and A.H. Meyer, 1939. Improvement in deniges colorimetric method for phosphorus and arsenic. 1st Eng. Chem. Anal. Ed. 1:136-139.

Viti R. and F Cinelli, 1993. Lime induced chlorosis in quince rootstocks: Methodological and physiological aspects. J. Plant Nutrition, 16(4): 631-641.

Wahome, K.P., T.M. Masarirambi and D.V. Shongwe, 2010. Evaluating different hydroponic systems for growth, flowering and quality of gladiolus (Gladiolus grandiflorus. Int. J. Agri. Bio., 12(5):649-654. 